\title{
Energy Management in RFID-Sensor Networks: Taxonomy and Challenges
}

\author{
Shaik Shabana Anjum, Rafidah Md Noor, Mohammed Hossien Anisi, Ismail Bin Ahmedy, Fazidah Othman, \\ Muhammad Alam and Muhammad Khurran Khan
}

\begin{abstract}
Ubiquitous Computing is foreseen to play an important role for data production and network connectivity in the coming decades. The Internet of Things (IoT) research which has the capability to encapsulate identification potential and sensing capabilities, strives towards the objective of developing seamless, interoperable and securely integrated systems which can be achieved by connecting the Internet with computing devices. This gives way for the evolution of wireless energy harvesting and power transmission using computing devices. Radio Frequency (RF) based Energy Management (EM) has become the backbone for providing energy to wireless integrated systems. The two main techniques for EM in RFID Sensor Networks (RSN) are Energy Harvesting (EH) and Energy Transfer (ET). These techniques enable the dynamic energy level maintenance and optimisation as well as ensuring reliable communication which adheres to the goal of increased network performance and lifetime. In this paper, we present an overview of $\mathrm{RSN}$, its types of integration and relative applications. We then provide the state-of-the-art EM techniques and strategies for RSN from August 2009 till date, thereby reviewing the existing EH and ET mechanisms designed for RSN. The taxonomy on various challenges for EM in RSN has also been articulated for open research directives.
\end{abstract}

Index Terms-IoT, RSN, RFID, WSN, Energy Management, Energy Harvesting, Energy Transfer.

\section{INTRODUCTION AND MOTIVATION}

$\mathbf{T}$ HE emergence of wireless communication protocols, efficient application based sensors, cheaper processors and ubiquitous computing had given birth to the evolution of IoT mainstream. According to IoT research, the quantity of internet-connected devices has transcended the number of users in 2011 and by 2020, internet connected devices are expected to amount to nearly 50 billion. IoT is referred to as a vast ubiquitous prevalence of network of things which are capable of interacting with each other and are capable of providing huge amount of data and information to the internet in the future. It is a pervasive computing based platform that circumscribes to a wide array of enabling technologies to extend their individual internet capabilities and bridge the gap between the real world and the virtual world of things. The IoT platform extends communication well beyond the traditional

S.S.Anjum,R.Noor,I.Ahamedy and F.Othman are with Faculty of Computer Science and Information Technology, University of Malaya, Kuala Lumpur, Malaysia.e-mail:(anjumjavid@siswa.um.edu.my),(fidah@um.edu.my) (ismailahmedy@um.edu.my),(fazidah@um.edu.my)

M.H.Anisi is with School of Computer Science and Electronic Engineering, University of Essex, Colchester, United Kingdom.e-mail:(anisi@ieee.org)

M.Alam is with Instituto de Telecomunicacoes, Aveiro, Portugal.email:(alam@av.it.pt)

M.K.Khan is with Center of Excellence in Information Assurance, King Saud University, Riyadh, Saudi Arabia.email:(mkhurram@ksu.edu.sa)

Corresponding authors - email:(fidah@um.edu.my) and (anisii@gmail.com) realm.

IoT can be defined as a paradigm where every day physical objects are connected together through Internet. The successful evolution of IoT vision, has paved way for computing portables and smart phones as an extension of past traditional scenarios. The evidence of such advancements can be witnessed in the blooming existence of 4G-LTE and Wi-Fi. Such a seamless integration of physical objects into the information network provides intelligent and ubiquitous services leading to a promising future in the fields of surveillance, health care, security, transport, food safety, object monitoring and control. The integration of both sensing and identification technologies with the aim to optimise the energy consumption as a whole, through energy harvesting from renewable energy resources, is the motivation of this research. The process of combining both identification and sensing technologies together is enabled by the IoT platform. The IoT researchers have urged that all entities in the future will have distinct characteristics for identification that can be connected with each other to create the IoT hub. Consequently, the aspect of communication will shift from being active solely between humans to communication between humans and virtual world, which will transform life. It has been effectively predicted that the evolution of pervasive computing and ubiquitous communications has made RFID and other sensing technologies such as WSN, the keystones of the future IoT generation. The progressive advancements of IoT will depend on the improved performance, especially in reliability and energy efficiency of WSNs. The reliable delivery of sensor data plays a vital role in the performance of WSNs for smart IoT environments. Radio Frequency Identification (RFID) and Wireless Sensor Networks (WSNs) represents most prominent dual technologies that have a wide range of applications. These dual ubiquitous computing based technologies have gained considerable attention in potential research and development fields. RFID applications include the supply chain management, manufacturing, search and rescue. On the other hand, WSN technology is used for sensor node deployment to monitor air pollution and battlefield surveillance. The integration of RFID and WSN has paved way for the evolution of RSNs. RFID is used to track or locate an object's identity in the absence of traces about the physical environment of the entity. WSN, on the other hand, are networks of small interconnected tools that are incorporated to collect information by discerning the habitual status of the surroundings like temperature, light, humidity, pressure, vibration and sound. These two technologies bestow extended capabilities, enhanced efficiency, cost effectiveness and eventually mend the gap between the real and the virtual 
world. The requirements for the development of RSN include accurate communication, reliability, energy efficiency, network maintenance survivability, tolerable latency and criticality of the application. Energy efficiency is considered to be one of the most attention seeking limitations because both sensor nodes and RFID tags are comprised of scarce resources. The RSN can, otherwise, be termed as Computational RFID (CRFIDs) or Wireless Identification and Sensing Platform (WISP) which adjoins the recognition capability of the RFID technology and the sensing/computing potency of the wireless sensors. The factual issue lies in the matter of periodical recharging of these WISP devices which puts forth the challenge of potential deployment of wide RSNs area consisting of mobile RFID readers and energy scarce WISP nodes. In order to make the integrated system efficient and reliable, the goal of a minimum possible energy consumption should be focused upon. Our contributions include (1) A better understanding of RSN with its basic working, architectural types and potential applications (2) Energy management concept, techniques and architecture in RSN and (3) Open issues, taxonomy and conclusion followed by future research directives.

\section{RFID-SENSOR NETWORKS}

The concept of integrating RFID and WSN has given way for the evolution of RSN. Figure 1 shows an architecture of RSN, hence bridging the gap between virtual and real world entities. Both RFID and WSN are reckoned to be as the two most significant enabling technologies of IoT. This integration provides newer perspectives to a wide variety of scalable, portable and cost effective applications. The assimilation of both RFID and WSN can eventually maximise the functionality and provide the means for an effective scope for pervasive computing aspects. RFID utilises the identification potential to trace the location of an object whereas WSN on the other hand, provides information about the physical condition of the object and the surrounding environment by enabling multihop communication. These two promising technologies when integrated together will result in extended capabilities along with portability, scalability and the reduction of unnecessary costs [1].

\section{A. Types of RFID-WSN integration architectures}

The two types of RFID tags are active tags and passive tags. The active tags use an external power source for charging its radio for communication purposes with the mobile reader. The passive tags utilised energy provided by the reader when it comes to the reading range of the reader by means of electromagnetic induction. The frequency range of active tags ranges at around $2.4 \mathrm{GHz}$ and for passive tags at under $100 \mathrm{MHz}$. The storage capacity for active tags is around $128 \mathrm{~K}$ bytes whereas for passive tags it is considerably lesser. The RFID readers are majorly classified according to the frequency in which they operate. The categorisation also depends upon the type of application in which the RFID readers and tags have been employed. The Low Frequency RFID operates in the range of $30 \mathrm{KHz}$ to $300 \mathrm{KHz}$ with a tag detection ranging

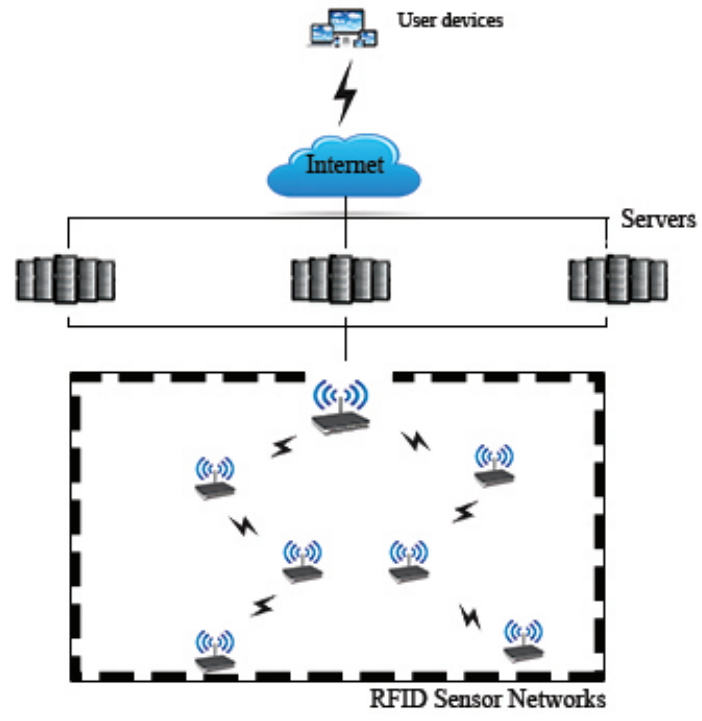

Fig. 1: Architecture of RSN

up to $10 \mathrm{~cm}$. But the High Frequency RFID operates in the range between $3 \mathrm{MHz}$ to $30 \mathrm{MHz}$ and is used for applications where the tag detection needs to be tracked for a distance of more than $10 \mathrm{~cm}$, which is considerably higher than the previous type. The Ultra High Frequency RFID readers are the most common and fast developing among all the types, because of its high operating frequency band of $300 \mathrm{MHz}$ to $3 \mathrm{GHz}$ and the tag detection range of about $12 \mathrm{~m}$. These types of RFID transponders are most receptive towards interference because of their high frequency range. The following Table I depicts the RFID frequencies and characteristics. There are four classes of integration that have been explored and validated by the researchers till date. They are i) integrating tags with sensors ii) integrating tags with WSN nodes and wireless devices iii) integrating readers with WSN nodes and wireless devices and iv) a mix of RFID and WSNs. Figure 2 shows the different kinds of RSN integration. The requirements for integrating RFID and WSN include factors adhering towards energy efficiency, reliable communication, accuracy and network survivability.

There are numerous research contributions in the literature that provide insights into the various types of architectures for integrated RFID and WSN. The RFID tags those which were embedded with sensors are classified into two major classes. The sensor tags which when integrated can communicate with RFID readers and the sensor tags upon integration are able to communicate with each other to form a network. The authors in [2] provide a survey on the latest research contributions, new products, patents and applications that integrate RFID with WSNs where elaborated information can be referred on different types of integration, its applications and technical challenges. When RFID tags are integrated with sensors, their communication capabilities are either limited or extended. There are active, semi-active and passive integrated RFID sensor tags which exhibit their own properties and have distinctive features from each other as explained in detail by authors in [1]. The four major classes of integration architecture have 


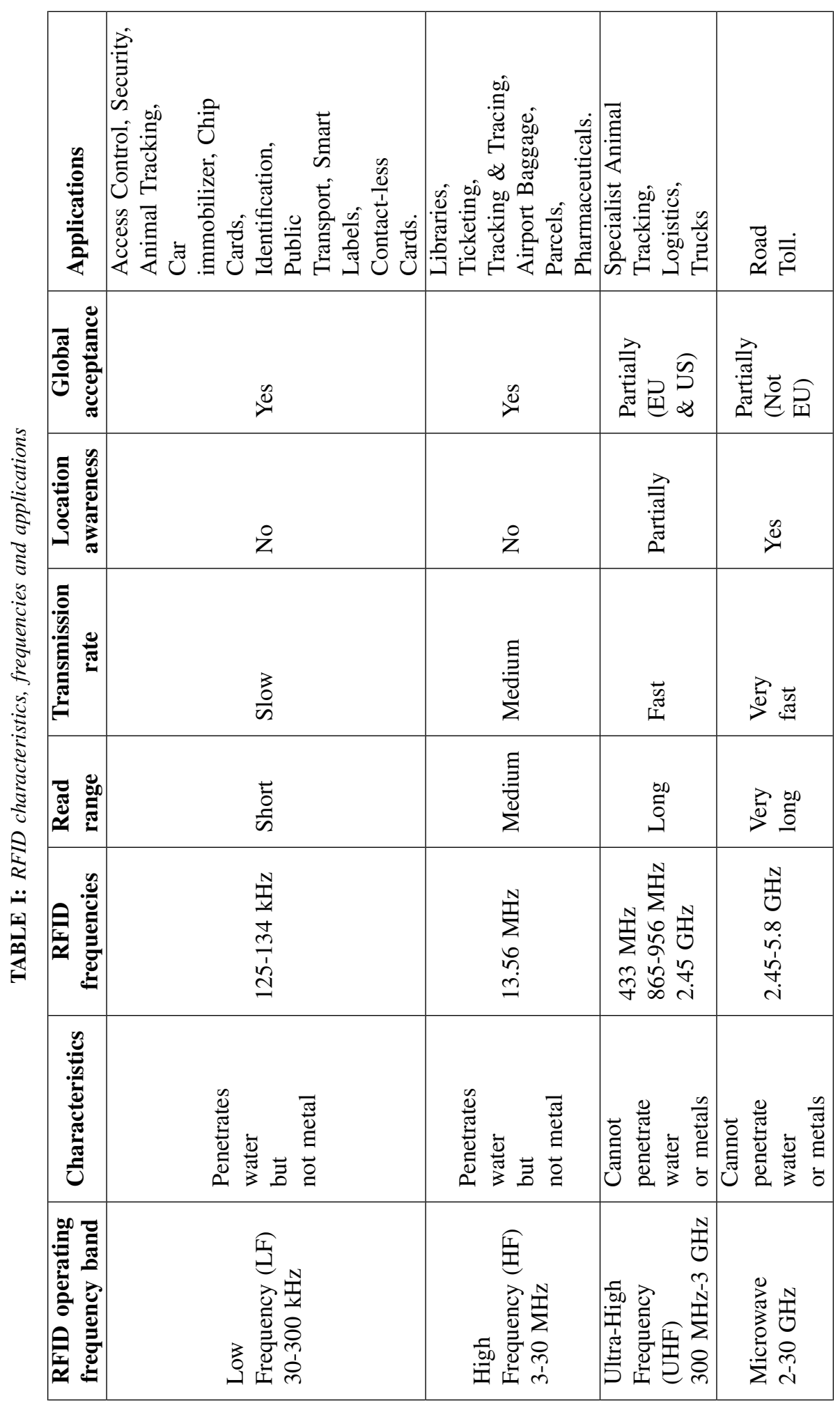




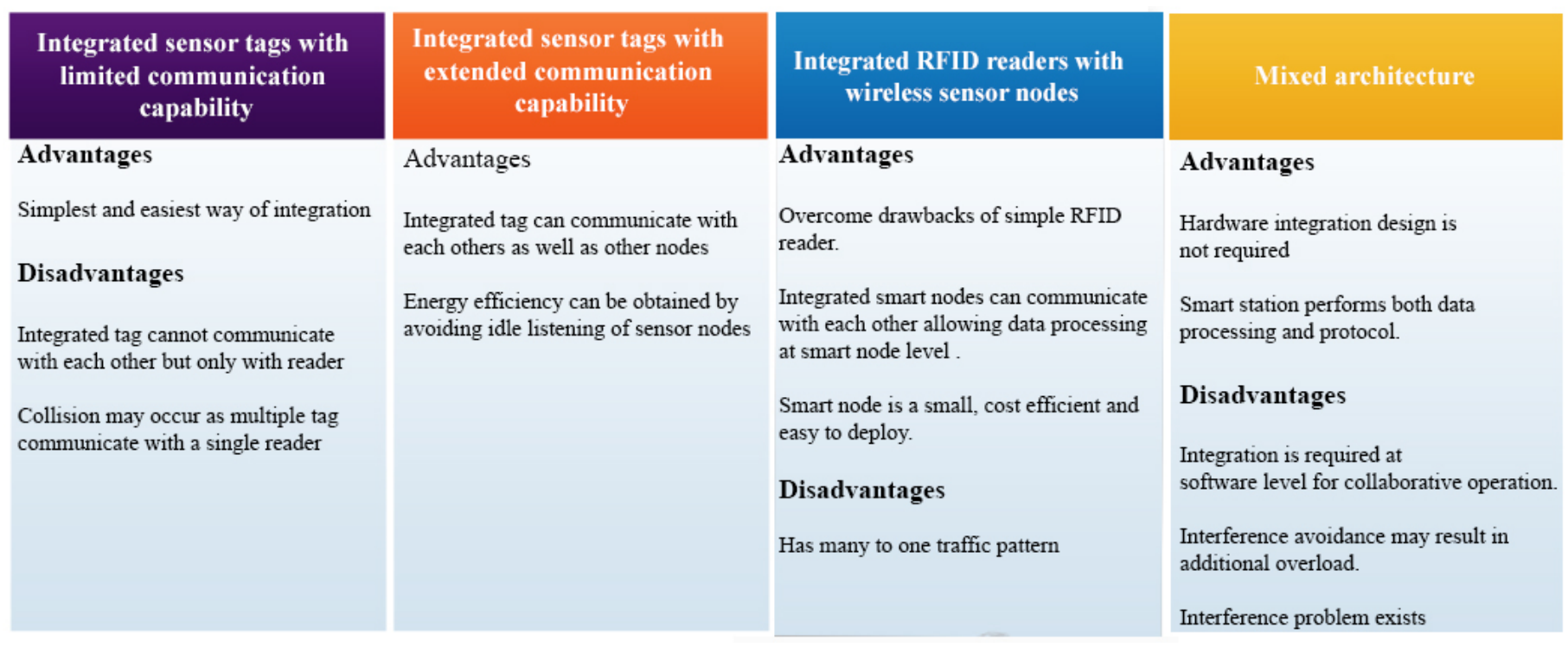

Fig. 2: Types of RFID-WSN integration architectures

their own advantages and disadvantages as depicted in Figure 2 but the sensor tags with extended communication capabilities are said to be superior among all the four classifications since it enables an energy efficient operation and has no shortcomings as recommended in [3], [4], [5] and [6]. Ahmed Jedda et al. [7] have investigated the interconnected target coverage problem in RSN. The authors have also surveyed on different approaches to solve the coverage and connectivity problem with the help of distributed algorithms. Wang et al. [8] have presented a five-layered architectural design to integrate WSNs and RFID systems. Bluetooth and ZigBee technologies are selected as the communication protocols for WSN to meet the requirements of large node density, coverage and cost factors. An Improved Cross Redundant Data Cleaning (ICRDC) algorithm is proposed in [8] and validated to alleviate the superfluous data in integrated WSN systems. Wang et al. [9] had proposed the EPC Sensor Network (ESN) architecture as a standardisation platform for heterogeneous integration of RFID and WSN. The ESN architecture is based on the Electronic Product Code (EPC) global architecture, which is the de facto international standard for RFID and Complex Event Processing (CEP) technology to handle huge quantities of events from distributed RFID and sensor readers in a real world scenario. The authors in [10] have proposed a novel data cleaning algorithm based on clustering mechanism where the cluster head eliminates the redundant equivalent data and forwards the filtered data towards the base station or the sink node. Fadi et al. [11] have proposed a new architecture for integrated RFIDs and WSNs to shelter an array of applications in a cost- effective manner based on Integer Linear Programming (ILP). The researchers in [12] have presented a chapter that gives a brief introduction on RFID and investigation on all literature work, patents, end user devices and operational utilisations that incorporate RFID with sensor networks.

Jin et al. [13] have provided insights into the basic organisation of sensor integration to EPC global network, a survey of applications and a relative background study to reveal the technological needs. The ontological perception and a role/interface based reference model, layout of roles and interfaces of sensor-integrated EPC global network have been presented. The researchers in [14] have highlighted the major discrepancies between RFID and WSN followed by its potential uses and a taxonomy discussion about integrated RFID-WSN systems. Jongwoo et al. [15] have urged that besides the popularity of current RFID and WSN, the present research lacks insights into a universal perception that is essential for ubiquitous computing. The authors have proposed in [15], a comprehensive standard framework for RSN based on EPC-Global standard architecture. The other relative research contributions that provide insights into the types of RFID/WSN integration and its potential applications have been described in [16], [17], [18], [19] and [20].

\section{B. Applications of $R S N$}

Majid et al. [21] have presented a module that is powered with radio and asymmetric wireless link employing ultrawideband radio system for RFID and WSN applications. The research has acknowledged that impulse-based Ultra Wide Band (UWB) is a hopeful and pivotal technology for RSN applications. Table II shows a number of RSN applications. Sajid et al. [22] have presented an integrated RSN system for smart homes such as tracking or tracing the whereabouts of a caretaker who arrives at home, for handling lighting, personalised music and to identify motion within an environment under surveillance. Reiner et al. [23] have proposed a sensor system that self-configures to a product specific supervision function based on data scanned by an RFID reader during consignment loading. Mobile software agents follow the shipping along the supply chain. They pre-process the huge chunks of sensor data and acknowledge only consequential changes to the freight owner. Ricardo et al. [24] have proposed a study on the evaluation of dynamic behaviour of 
TABLE II: Applications of RSN

\begin{tabular}{|c|c|}
\hline $\begin{array}{l}\text { Application } \\
\text { Type }\end{array}$ & $\begin{array}{c}\text { Technologies } \\
\text { employed }\end{array}$ \\
\hline UWB Communications [21] & $\begin{array}{l}\text { Passive CMOS tag with asymmetric } \\
\text { link }\end{array}$ \\
\hline Smart Homes [22] & Alien technology ALN-9534 tags \\
\hline Freight loading [23] & UHF readers, Intels strongARM \\
\hline $\begin{array}{l}\text { Cold chain monitoring and } \\
\text { tracking [24] }\end{array}$ & $\begin{array}{l}\text { Wireless ZigBee motes: } \\
\text { IRIS,NLAZA, turbo tag 702-B, turbo tag } 700\end{array}$ \\
\hline $\begin{array}{l}\text { Urban sensing through vehicles } \\
\text { [25] }\end{array}$ & $\begin{array}{l}\text { TGS } 2201 \text { sensor, TGS } 2442 \text { and TGS } \\
2600 \text { sensors }\end{array}$ \\
\hline Credit card system [26] & $\begin{array}{l}\text { Clustering algorithm, trusted } \\
\text { platform module }\end{array}$ \\
\hline Structural Health Monitoring [27] & PetriNet toolbox \\
\hline Electrical industry [28] & TGIS. NDIS, ZigBee \\
\hline Health Monitoring [54] & HRW, GRFID, HSN \\
\hline $\begin{array}{l}\text { Tracking and tracing } \\
\text { identification [56] }\end{array}$ & SNAP nodes, RPC functions \\
\hline $\begin{array}{l}\text { Supply chain Management systems } \\
\text { [55] }\end{array}$ & MDI,EPCIS,RESCUEIT \\
\hline Smart Parking system [29] & UHF, RFID, IEEE 802.15.4 \\
\hline Healthcare facilities [30] & WBAN, BSN, ISM-RF, CDSS. \\
\hline Logistics [32] & RFID, WSN, Statistical modelling \\
\hline Supply chain Management [33] & JIT, SOA,CSCM environments \\
\hline SmartExhibition [32] & $\begin{array}{l}\text { RFSenMote, ZigBee based RFID } \\
\text { network, EEPROM. }\end{array}$ \\
\hline
\end{tabular}

wireless sensors on integrated systems. In [25], the researchers have proposed a novel technique called EnvironSense which is relied upon RFID magnetic induction to detect the air contamination through environment based sensors equipped near the exhaust of the vehicle and transmit the relevant data back to the driver through feedback. Rohit et al. [26] in their research contribution presented an integrated RSN to frame a smart and error free RFID credit card system. In [27], the authors have presented a study that demonstrates the algorithm for Structural Health Monitoring (SHM), by employing the RFID with sensor-tags integrated system in order to gain insights into the communication aspects. Kim et al. [28], have developed a prototype system for testing the probability of electrical industry application using RFID/WSN to manage the electric facility. The researchers in [29] have presented a Smart Parking System (SPS) based on the integration of Ultra High Frequency (UHF) Radio Frequency Identification (RFID) and IEEE 802.15.4 technology to ensure robustness and interoperability of Vehicular Adhoc NETworks (VANETs). Shaftab et al. [30] have proposed a hierarchical security management technique that uses biometric data, e-health card particulars or RFID tag for patient or doctor authorisation to handle healthcare services. The research article in [31] had presented an integrated RSN architecture to be applied on animal and habitat monitoring applications. Sobhi et al. [32] have proposed an integrated model system in which RFID capabilities are combined with WSN technology to serve as a reliable decision making platform when products, materials and information are moved across the supply and demand chains. Zulkifli et al. [33] have developed seamless and logically integrated information management architecture that can produce information about agriculture using IoT protocol and validating the updates to project stakeholders for the effective decision making process.

\section{ENERGY MANAGEMENT IN RSN}

In RSN, the main advantage lies in the fact that sensors do not need to contain batteries for transmission of sensed data. In order to maintain the energy levels to remain in an operational state for sensing and processing of data, there has to be effective EM techniques. The RSN node which comprises of passive RFID tag and sensor has a distinguishing feature of getting recharged through the RFID reader when it is in closer proximity range. The node acts as a storage device when it is away from the range of the reader. The concept of ambient backscattering is applied on the WISP hardware between the node and the mobile reader. The other types of RFID tags such as active and semi-active do not rely on backscattering modulation for operation but depend solely on energy storage devices such as batteries and capacitors installed at the time of the initial set-up. Therefore, they involve an increased cost and have limited lifetime when compared to passive tags which gets activated through the RF field emitted by the readers [34].The most prominent feature that determines the performance and throughput of RSN is the ability of the reader to read the data from the RSN nodes. This reading range at which the reader communicates with the nodes depends upon two major aspects. First, is the energy rate at which the node gets activated to be in an operational state (tag reactivity) and second, is the power required by the reader to detect the backscattered RF signal by the node in a correct manner (reader responsiveness). EM addresses the issues pertaining to these two most vital aspects to ensure the performance and lifetime of RSN. The two main techniques for EM in RSN includes EH for tag's reactivity and ET rendering to reader's responsiveness. Figure 3 shows the strategies of EM in RSN pertaining to EH and Energy Storage (ES) for wireless recharging.

EH through RF signals allows the wireless devices to harvest energy from RF signals for data processing and information 


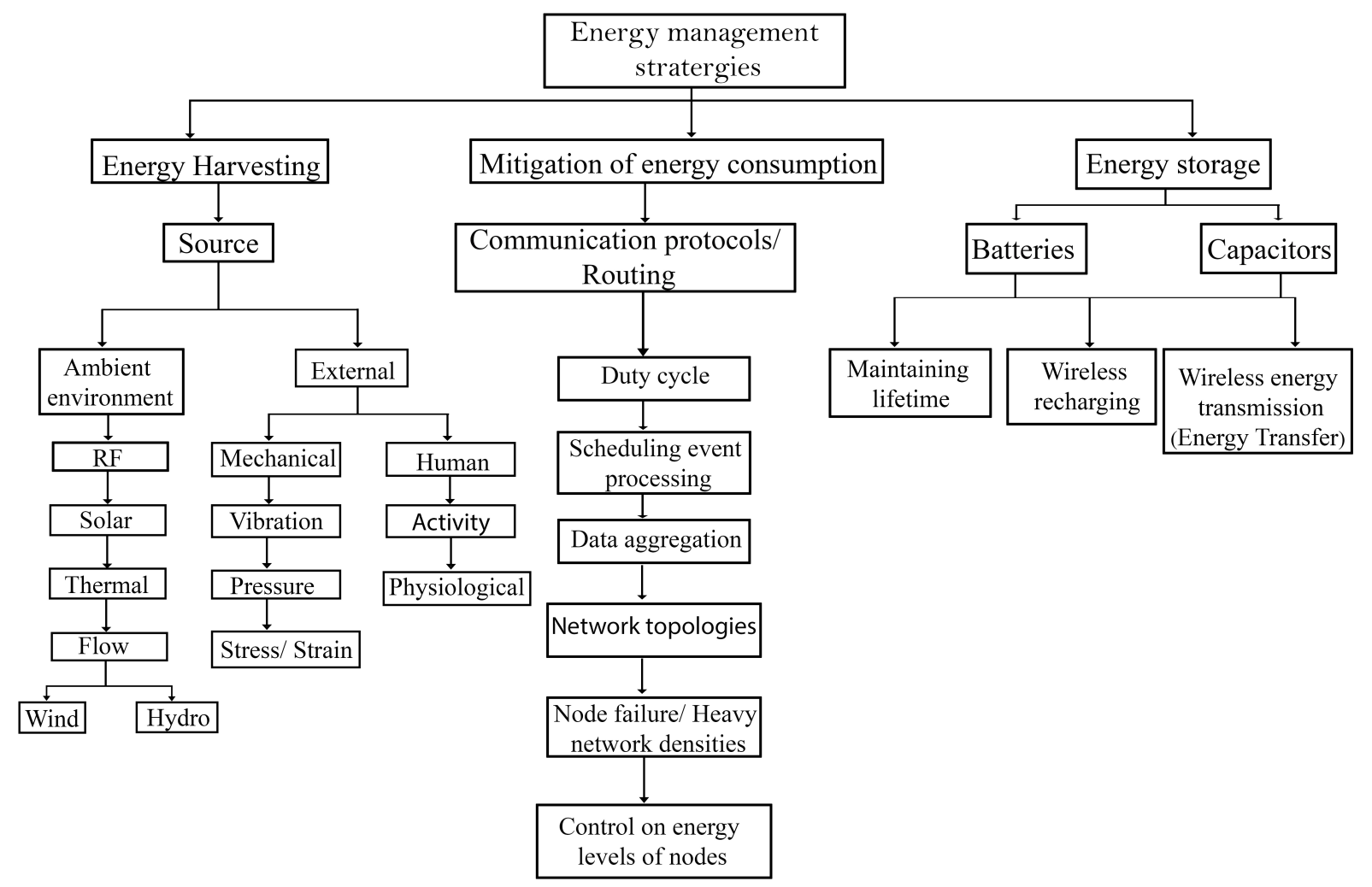

Fig. 3: Taxonomy of Energy management strategies in RSN

delivery [35]. Here, EH refers to the process of utilising the stored energy efficiently without relative energy loss or leakages. On the other hand, ET refers to the transmission of energy from a RF energy source to power scarce node over dedicated communication channels in data link layer. The literature has numerous contributions in the field of RF harvesting and transfer for WSN. The authors in [36] have presented their contributions on admission control problem and scheduling of data for a Backscatter Sensor Network (BSN). Figure 4 depicts the BSN model and the working architecture of typical backscatter communication through the gateway. BSN comprises of many wireless senor nodes which are designed to transmit informaion to a gateway node as shown in Figure 4(a). On contrary to the traditional WSN, in BSNs the sensor nodes communicate to the gateway for data collection, by transmitting RF signals through the transmitter antenna (Tx). The sensor on receiving the RF signals, reflect and backscatter these signals to the gateway again by switching the states of operation of RF impedance circuit, connected to its relative antenna as depicted in Figure 4(b). The controller handles the switch circuit of the sensor by generating sequence of 1's and 0's bits. The gateway node employs the reciever antenna $(\mathrm{Rx})$ to receive the signals from sensor. The gateway can extract data from sensor with the use of analog-to-digital convertor (ADC) and a demodulator. The readers can gain an in-depth background knowledge about BSN in [36].

EM in WSN includes factors such as the reduction of power consumption by Cluster Heads (CHs) in order to prolong the network lifetime. The number of clusters in the network also determines the overall energy consumption in addition to load distribution and communication overhead. The researchers, in their contributions, so far have articulated that the methods by which energy can be managed in any sensor networks could be threefold: They are either by mitigating the energy consumed by the nodes, harvesting or conserving the energy from renewable energy sources and transferring of energy over a minimum communication overhead and interference avoidance. In the Wireless Rechargeable Sensor Networks (WRSNs), recent studies have focused on identifying a suitable possible configuration or path movement for the Mobile Charger (MC) in order to prolong the network lifetime and hence to enhance energy efficiency [37]. These contributions focus on the aspects such as the distribution of energy between the MC and the sensor nodes, the level to which each node is to be charged and also the best possible configuration of $\mathrm{MC}$ to charge the nodes. EM is foreseen to play a major role in WSN and the fundamental issue lies in the fact that these networks are powered by batteries and capacitors which are bound to have a limited lifetime. The energy optimisation problem is formulated by authors in [38] by employing multiple mobile sinks for data collection in WSN. The concept of Amplified Backscattering via $\mathrm{EH}(\mathrm{ABEH})$ is proposed in [34] with the focus on allowing the amplification of the backscattered signal to improve the read range of conventional passive RFID tags. 


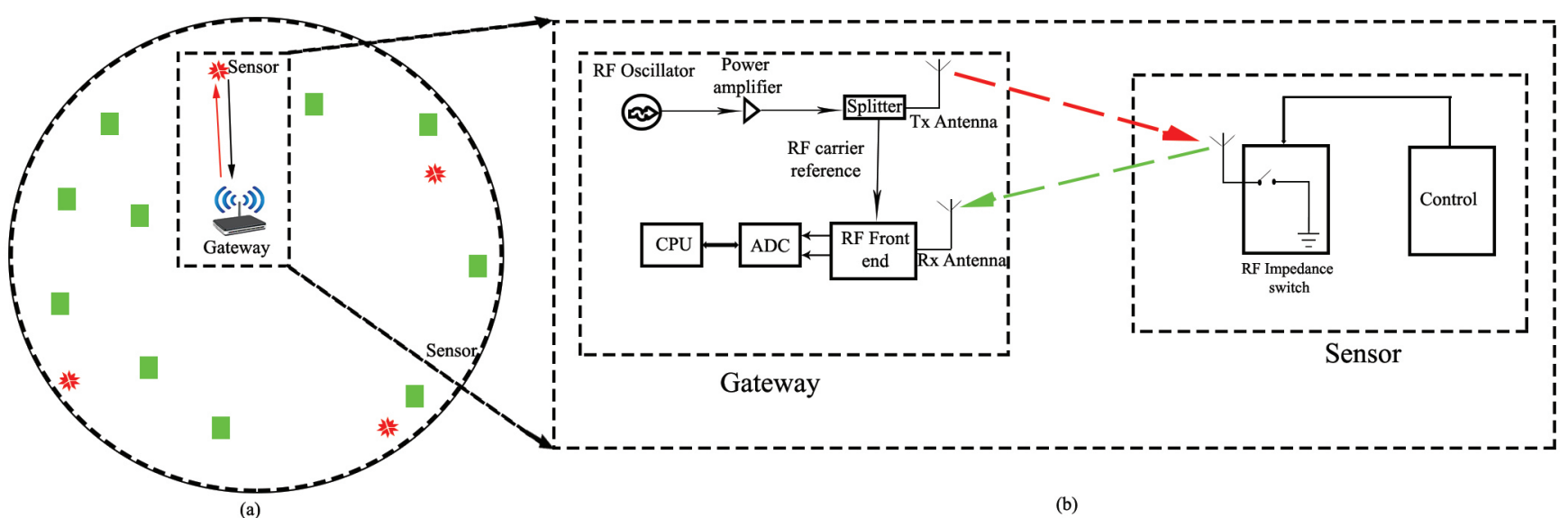

Fig. 4: BSN model and backscatter communication architecture

Figure 5 illustrates the block diagram of RFID ABEH sensor. The dashed border shows the new components in contrast to the classical passive RSN nodes. The challenge in this scenario was to ensure a workload balance between the mobile sinks and also to guarantee optimal energy consumption among the sensor nodes via dynamic control on the trajectory of mobile sinks. The research work in [39] provides a Compressed Sensing (CS) framework for data acquisition in the information systems comprising of WSNs and IoT. This CS based framework has proven to be a promising solution for energy saving and preventing communication overhead in reconstructing the sparse or compressible data.

Another class of sensor networks which has overcome the drawback of limited lifetime of energy storage devices are BSNs. These classes of networks rely upon RF energy harvesting techniques to remain in an operational state for sensing and processing data. Differing from conventional WSNs where the sensor nodes transmit data directly to the gateway, in the case of BSNs the gateway transmits RF signals to the sensors which upon reception, are backscattered to the gateway to get processed for the modulated information [36].

RSN comprises of miniature sensor nodes which are deployed to gather information on a particular object or area. The ability of these networks to monitor remote and hostile environments along with tracking capabilities has attained significant attention for research in the past few years. There are also technical challenges to be met in order to maintain efficiency and reliability of the connected network and active nodes participating in the communication process. These challenges include resource constraints, transmission range, storage, and energy consumption followed by battery power of the sensor nodes. The existing solutions do not consider how to optimally allocate transmitted power and provide power splitting ratio to minimise energy efficiency. The most challenging aspect lies in estimating the periodicity and magnitude of the harvestable source and the decisive factor for the avoidance of energy exhaustion before the next recharge cycle. The process of conserving energy from renewable energy resources is termed as Energy Harvesting. The sensor networks when combined with identification capabilities need more energy to communicate and transmit data over dedicated channels. This need has made many researchers to develop methods for maintaining the energy levels of the sensor nodes thereby focusing on the goal of the overall network performance and reliability. The design of the WISP device, its relative power/energy budget and bidirectional communication capabilities are discussed

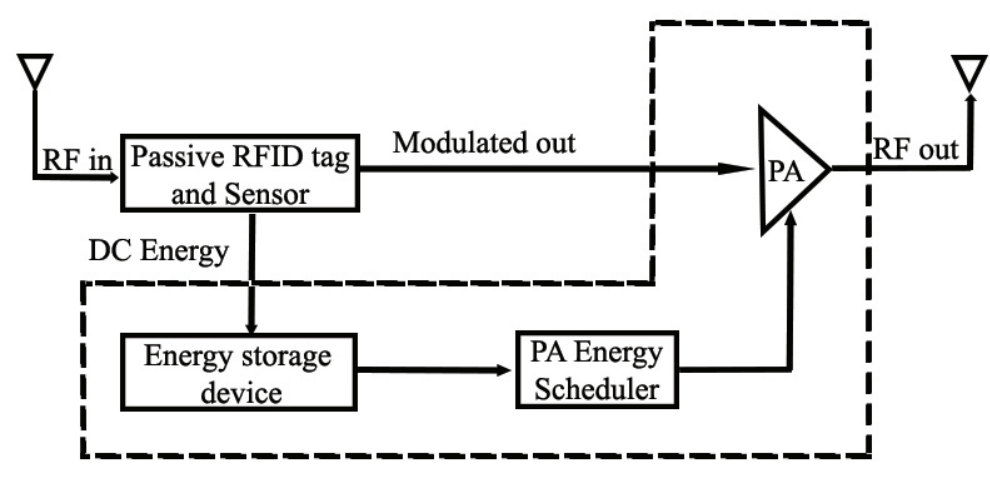

Fig. 5: Block diagram of RFID ABEH sensor tag 


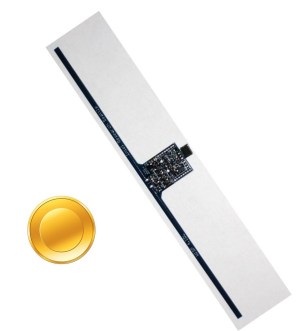

Fig. 6: WISP platform

in [40]. Figure 6 shows the WISP platform in compliance with the EPC Class 1 Generation 2 (C1G2) protocol. The need for energy efficient routing protocols is a necessity for increasing the lifetime of the network and to prevent node failure. The operation and maintenance of sensor nodes in an intimidating environment and also the presence of error prone communication links exposes these networks to low energy levels, thereby hindering the overall network performance and throughput. The network infrastructure of heterogeneous devices in IoT contexts are equipped with sensors, processors, wireless transceivers and energy resources for data transmission and monitoring activities. One of the dominant hurdle to implement such interoperable networks is supplying of adequate energy for network operation without conceding on Quality of Service (QoS) [41]. Hence, it is important to increase the energy efficiency of the connected devices in the sensor network. The authors in [42] have highlighted that numerous energy efficient protocols have been designed to extend the lifetime of the sensor nodes. In traditional WSN, the incorporation of mobility technology with traditional static sensor networks, guarantees a new elucidation that balances energy consumption among the sensor nodes and eventually extends the lifetime of the network. The power management protocols can be employed either as independent sleep/wakeup protocols running on top of a MAC protocol (at the network or application layer), or strictly integrated with the MAC protocol itself [43]. A standard protocol needs to be used for communication in sensor networks. Emerging communication standards such as IEEE 802.15.4 are being used in WSN as an underlying protocol for building other standardised communication protocols such as ZigBee and 6LoWPAN [44]. A critical performance principle in backscatter modulation-based RFID sensor networks is the distance in which a RFID reader can consistently communicate with passive RFID sensors (or tags).

\section{A. Energy Harvesting in RSN}

The process of effectively harvesting energy from renewable energy sources such as solar, vibration and radio frequency is termed as energy harvesting or energy conservation. RSN employs the concept of harvesting energy via the RF signals emitted from a RF energy source. This type of power conservation technology enables proactive and dynamic energy replenishment. The authors in [45] have proposed a hybrid EM system which has proven to stabilise the power delivered to the load, using the novel scheme of magnetic, thermoelectric and vibrational EH system. The issue of increasing the network lifetime of WSN is studied in [46] where CHs collect, aggregate and send the data directly to the sink serving as relay nodes equipped with $\mathrm{EH}$ capabilities. The state-of-art solutions in the aspect of solar cum electromagnetic EH and wireless power transmission has been delivered in [47]. Recent studies have found that balancing both data transmission and transfer of energy over wireless medium puts forth a significant challenge on the working pattern of wireless networks. Eventually, both the aspects cannot be optimised or maximised simultaneously that raise the need for re-evaluating the design pattern of existing wireless networks [35]. In the process of $\mathrm{EH}$, the amount of harvested energy relies on the total power transmitted, the RF signal wavelength and distance between harvesting node and the RF energy source. The harvested power from RF transmitter can be calculated using Friis equation 1 in free-space model as,

$$
P_{R}=P_{T} \frac{G_{T} G_{R} \lambda^{2}}{(4 \pi d)^{2} L}
$$

where, $\mathrm{d}$ is the distance between the transmitting antenna and receiving antenna, $P_{R}$ is the power received, $P_{T}$ is the power that is transmitted, $\lambda$ is the wavelength of the RF signal, $\mathrm{L}$ is the path loss factor, $G_{T}$ is the antenna gain of the transmitter and $G_{R}$ is the antenna gain of the receiver. On the other hand, the RF harvested power from transmitter as per equation 2 for two ray ground model is derived from,

$$
P_{R}=P_{T} \frac{G_{T} G_{R} h_{t}^{2} h_{r}^{2}}{(d)^{4} L}
$$

where, $h_{t}$ and $h_{r}$ are the heights of the antennas at the transmitting and receiving side respectively. The researchers in [48] have designed, fabricated and tested an antenna of differential microstrip with the gain of $8.5 \mathrm{dBi}$ and a bandwidth of 135 $\mathrm{MHz}$ for usage in RFEH applications. This is calibrated to be employed either for centralised or differential configuration, and serves as a front-end module for RF transceivers. One interesting research contribution involves the innovation of two port RFID tag antennas integrated with RFID chips for dual $\mathrm{EH}$ in order to support the operational state of MCU and sensor as well as increasing the read range of RFID tag [49]. The article in [50] focuses on the resource allocation drawbacks in Radio Frequency Energy Harvesting (RFEH) networks (RF-EHNs). The authors have described the optimisation of operational policies in order to enhance the throughput of the RFEH node and also to provide research directives with regard to spectrum sharing and cooperative sensing, energy trade-off, distributive energy beam forming and interference management. The concept of EH in RFEHN is either Infrastructure based (IS) or Infrastructure Less architecture (ISL). A basic IS based architecture of RF-EHN is depicted in Figure 7. It comprises of three modules- RF energy sources (RF energy transmitter/ambient RF sources), information gateway (base station, routers) and network devices. The information gateways communicate with the network nodes for user queries 
Infrastructure based Architecture

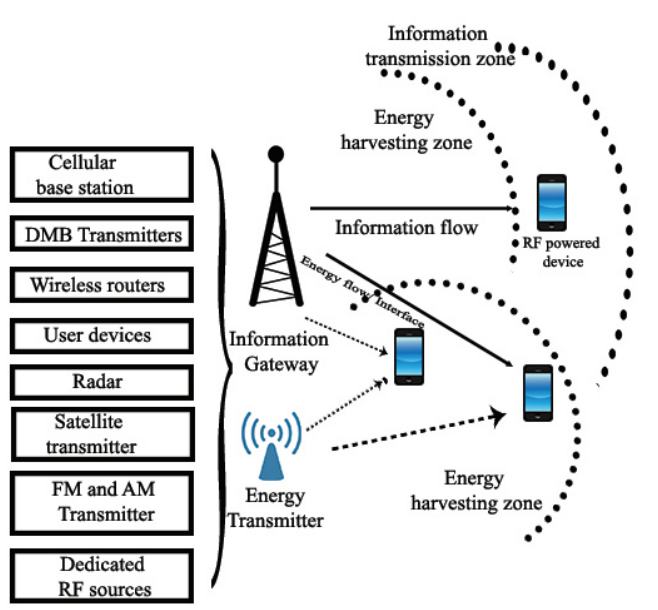

Infrastructure less Architecture



Fig. 7: Architecture of RF-EHNs

or requirements. The gateway nodes and RF energy sources are equipped with consistent electric supply, whereas network devices conserve RF energy from ambient resources. As shown in Figure 7, the dashed arrow lines depict energy flows and the bold lines represent information/data flow. The dashed curves represent the $\mathrm{EH}$ zone and data transmission zone of the information gateway. The ISL architecture is similar to IS, except for the fact that network nodes can communicate with each other directly [35]. The curse- of- model issue has been aimed to be avoided by the adoption of learning algorithms for performance optimisation in BSNs [36].

The harvested energy transfer as well as data transmission scheduling issue have been formulated as a Markov decision model. A comprehensive taxonomy of various EH concepts as well as energy prediction models that have the capability to enhance the harvested energy rate in WSN has been presented in [51]. $\mathrm{EH}$ in simpler terms is defined as the potential of collecting and efficiently utilising the ambient energy from sources such as RF signals, thermal energy, mechanical vibration, kinetic motion and so forth [52]. The research contribution in [53] has a Bluetooth Low Energy (BLE) beacon that functions on energy harvested from dual ISM-band RF energy sources with assistance from photovoltaic sources. The emergence of wireless charging techniques delivers a more flexible and promising way to solve the energy constrain problem in Wireless Rechargeable Sensor Networks (WRSNs). Although extensive research has been conducted on wireless charging algorithms, majority of them have focused either on passively replenishing energy for nodes with inadequate energy or providing event driven duty cycles according to the energy needs [54] and [55]. When the energy of a sensor node is exhausted, it will no longer fulfil its role in the network unless either the source of energy is replaced or some harvesting mechanism is induced to bridge the energy gap [56]. The prevailing solutions utilise energy source powered by batteries in sensor nodes of RSNs, but are associated with many draw- backs, like chemical leakages, extreme weather conditions and limited energy density. The class of networks called Industrial Wireless Rechargeable Sensor Networks (IWRSNs) has been explored by many researchers in the recent past where the routing is either grid based or clustered. The focus of IWRSNs is to solve the energy recharging problem in a proactive way [57]. Energy Harvesting can also be beneficial in asset tracking where the smartphone capabilities are embedded with traditional asset tracking techniques [58] for example, APL and WOLF. These systems have proven to detect almost all the assets and save energy consumed by the smart phones. The focus on minimising the energy usage in smart buildings had been aimed by authors in [59], where a Smart Personalised System for Energy management ensures energy optimisation and conserves energy through renewable resources. The applications such as environmental monitoring and surveillance suffering major limitations such as target coverage due to which the sensor network lifetime is eventually hindered. Hence, the past decades have witnessed several research contributions for increasing the network lifetime and maintaining energy efficiency pertaining to address the issues such as target coverage, sensor connectivity and network fault tolerance [60]. The primary concern being the network lifetime and energy efficiency, several reviews and taxonomies exist in the literature for energy conservation in WSN [61], [62] and [63] whereas it still lacks comprehensive surveys about energy harvesting in RSN. This reason seems to be the sole purpose for writing this review article. Furthermore, several authors have developed varying concepts for energising the sensor networks through energy harvesting [64], [65] and [66]. The production management sector has been envisioned as an area where IoT plays a vital role in monitoring the energy consumption [67]. The focus of this research has also been extensively extended in the investigation of feasibility concerns for IEEE 802.11 for energy harvesting of low-power sensing applications [68]. Moreover, Energy Harvesting has also found 
to be beneficial in Nano medical applications using BANNET and macro- scale healthcare monitoring systems [69]. There is a significant amount of research contributions for solar energy based harvesting as well to ensure robustness and minimal cost of the network [70] and [71].

\section{B. Energy Transfer in RSN}

The researchers in [72] had claimed to have developed the first wireless ET protocol for heterogeneous frequency in RFEH and have improvised with about $59 \%$ higher duty cycle and $66 \%$ average network throughput performance over classical CSMA MAC protocol. The different aspects of design issues in RFEHNs are design of the circuit, networking using single hop, multi-antenna and cooperative relaying techniques followed by communcation protocols as depicted in Figure 8. Low power transfer for energizing the mobile terminals in wireless communication systems have been focussed upon with the progress in RFEH circuit. Single hop and multiantenna networking utilizes harvest-cum-transmit protocol for energy transfer and RF charging. RF signals have been employed for both conserving energy as well as transferring data as in Simultaneous Wireless Information and Power Transfer (SWIPT) technique developed by authors in literature. SWIPT delivers both RF energy and wireless information dynamically. However, it has the issue of consequent correlation between transmitted data and energy transferred. RF powered cognitive radio networks also face the trade-off on the design issues with respect to spectrum sensing and bandwidth management. MAC communication protocol in RF-EHNs needs channel access for information processing and data transmission. The dynamic nature of the communicating nodes makes the time taken for harvesting energy distinct and unpredictable. The MAC protocol based on CSMA/CA called as RF-MAC is developed for optimization of delivery rate of RF energy [35]. The ALOHA$\mathrm{Q}$ protocol employs Q-learning mechanism in a frame based ALOHA as an intelligent strategy for slotted selection enabling the feature of perfect scheduling from random access [73]. The results of ALOHA-Q have outperformed slotted ALOHA with respect to network throughput, data delivery delay and energy efficiency. The researchers in [74] have showed that it is possible to reutilize the existing Wi-Fi infrastructure to provide internet connectivity to devices that runs on RF-power. A novel communication system called Wi-Fi backscatter is presented that connects RF-powered devices to the internet. A study on EH network which consists of a Base Station (BS) and multiple EH Users (EHUs) is described in [75] where the BS broadcasts RF energy to EHUs on the downlink and the EHUs in turn sends data concurrently to BS over the uplink EH MAC for WSN operation in inaccessible and hostile locations. The functionalities of RF-powered WSN in a downlink SWIPT system through RF transmitters via ambient harvesting using stochastic geometry method has been analysed in [76]. The authors in [77] have investigated on the RF-DC conversion efficiency in an RF harvesting circuit when it is calibrated by digitally modulated signals. It was derived that this conversion depends upon the rate of modulation through the output filter of the rectifier formed through the capacitor and load value.
The Markov decision framework based optimisation solution has been proposed in [36] for fetching the best available data collection policies for the gateway in BSN. The ET and scheduling in the transmission of data process have been formulated to mitigate the data delivery delay along with the loss of packet issue. The researchers in [78] have presented two novel communication schemes for backscatter communication networks. These schemes enable the RFID tag to communicate with each other over a wide area of networks. The runtime for CRFID is termed as Dewdrop in [79] which is energyaware and makes efficient usage of the harvested energy by balancing and scheduling the task demands relative to the time of operation and residual energy levels. Buettner et al. [79] have stated about the dewdrop implementation in their research contribution. There are two major techniques: Low power wake-up and Low-power voltage sampling mechanisms for the implementation of dewdrop. Basically, the threshold that the device has to wake up at has no hardware mechanism. The charging mechanism for dewdrop has exponentially adapted polling intervals that is light weight and accurate. On the other hand, waking up to sample voltage invokes considerable amount of energy consumption. Hence, the ways for reducing it is either to do waking up less often or reducing the voltage sampling energy cost by a factor of 4 . Figure 9 and 10 shows the comparative performance metrics of dewdrop and hardware mechanism for two different tasks- Light (only sensing) and Heavy (sensing and returning back a value to the reader). Figure 9 shows the execution rate versus distance for the two tasks during two different run times. One of the run time is dewdrop and the other is very efficient but inflexible hardware mechanism (which is the state-of-the-art before dewdrop). It can be seen that the task rate of dewdrop nearly matches with hardware mechanism at a distance of $1.5 \mathrm{~m}$ for light program tasks. Dewdrop provides task rate of $60 \%$ per sec when compared to $65 \%$ per sec by hardware mechanism at a distance of $1 \mathrm{~m}$ from the reader for light program tasks. Conversely, it is evident that for heavy program task, hardware mechanism doesn't function at a distance of $2 \mathrm{~m}$ to $4 \mathrm{~m}$ from the reader when compared to dewdrop that falls smoothly by using scarce energy efficiently. Therefore, dewdrop doubles the range for heavy task. Figure 10 depicts the operating point by the dewdrop at a distance of $1.5 \mathrm{~m}$ and $3 \mathrm{~m}$ for heavy program task and $1.5 \mathrm{~m}$ for light task respectively. The best operating wake-up voltage found by dewdrop adaptively was within $0.1 \mathrm{~V}$, using exhaustive search and wake-up mechanism. Hence, it shows that dewdrop achieves better than $90 \%$ of maximum operating task rates for all distances. Therefore, this contribution drives the fact that conversely from traditional RFID tags, RFID based WRSN needs to recharge its stored energy to a level which makes these nodes functional for sensing, computing, processing and communication capabilities [80]. The energy recharging requests of sensor nodes must be sent to the mobile charges or energy source through wireless communications, which may be lost due to the changing network conditions. On the other hand, network densities also play a major for energy request. The more number of nodes, the load of energy recharging workload increases, hence decreasing the throughput and performance of the network [81]. The parameters to 


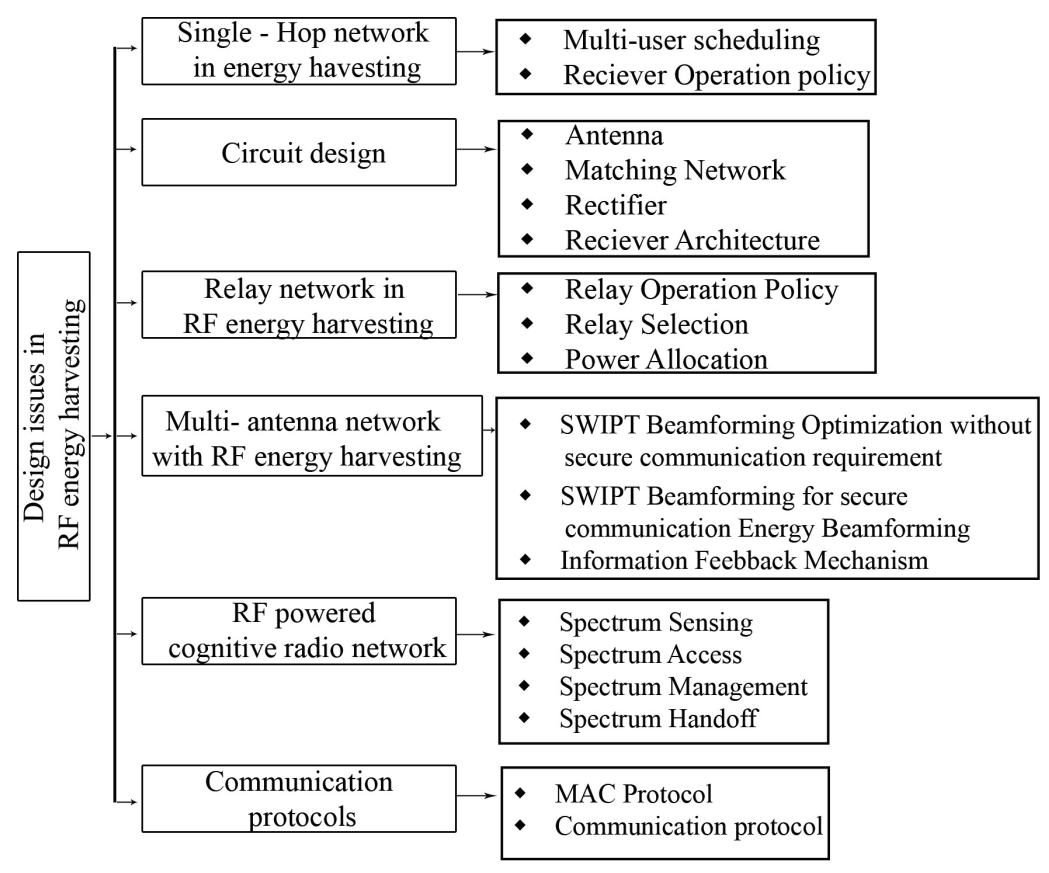

Fig. 8: Taxonomy of design issues in RFEHNs

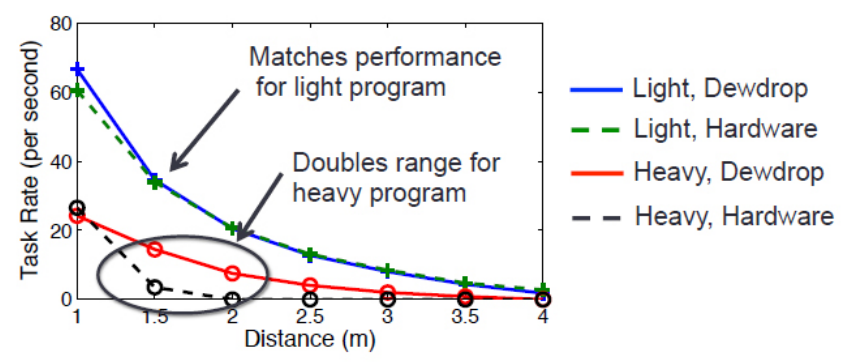

Fig. 9: Usage of Residual Energy by Dewdrop

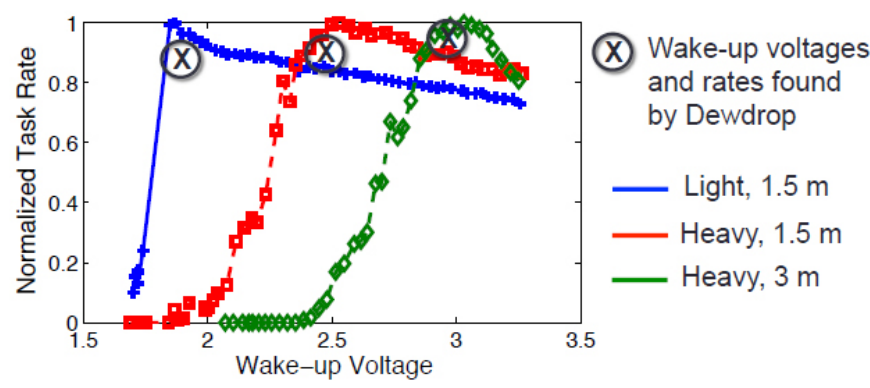

Fig. 10: Wake-up Voltage Vs Task Rate by Dewdrop

be considered are request delivery time, recharging time and travel time of the requesting sensor node. The on-demand energy recharging problem for RSN has to be analytically evaluated on the pretext of overall network throughput and energy efficiency that has not been addressed in literature. The drawback of effective wireless energy transfer and request in
RFID sensor networks still needs to be addressed between cooperative and clustered nodes. The major challenge in the network with wireless energy transfer is to decide the amount of energy to be transferred to the nodes [82]. The process of transmitting wireless energy not only requires an increased energy consumption by the access points, but also conceives a strong intervention to other networks sharing the same platform and their respective duty cycles. To overcome this expense of energy, the access point may recharge the nodes based on their wireless energy demand. The communicating nodes have to make the decision for energy transfer across wireless medium with regards to the charged cost and attainable data transmission conduct, from the access point. The existing solutions that are discussed in [81] focuses on non- cooperative scenarios for wireless energy transfer and requests, where the nodes can comparatively request for wireless power transfer for an active access point and use that energy for data packet transmission. Unfortunately, the issue of intervention management due to wireless energy transfer was not taken into consideration by the authors. Zhenping et al. proposed a distributive event-triggered interaction for time synchronisation so as to curtail the energy consumption in WSNs [83]. Ivan et al. proposed a co-operative solution to efficiently handle energy conservation and data collection in the wide areas of RSNs that are under heavy network densities [82]. Constantinos et al. proposed three novel routing protocols for efficient charging and also to address the vital limitations of efficient wireless energy transfer in WRSN [84]. The more attention seeking issues for energy transmission lies in the fact of quantifying the energy of each communicating node and the dynamics of communication when the nodes are in a sleep state. There are relatively fewer research contributions that are related to these aspects in the literatures 
till date. Fadi et al. introduced an architectural framework for node employment and fault delay tolerance in RSNs under the IoT standardisation [85]. Liguang et al. developed a mathematical model (OPT- $t$ ) which is dependent on time for locating conjointly a wireless charger and a mobile base station on the same spectrum [86]. An optimisation model has been formulated to derive the optimal policy for each mobile sensor node to capture the cooperation techniques and strategies about recurring coalition evolution [87]. Adelina et al. proposed four new communication protocols for efficient energy recharging and wireless energy conservation [88] while the authors in [89] have designed energy based and spectrum dependent MAC protocols for Wireless Nano Sensor Networks (WNSNs). The aim is to attain considerable network throughput and optimal lifetime of channel bandwidth access by mutually optimising the harvestable energy and power consumption processes in Nano sensors. Sindhu et al. had developed an efficient energy distribution algorithms, Q-learning algorithm, with exploration mechanisms based on the e-greedy method as well as Upper Confidence Bound (UCB) [90]. An energy consumption model based on energy consumption analysis for WNSNs by unitedly reckoning the energy consumption of both sender and receiver has also been developed and implemented [91]. Haipeng et al. proposed the approximation algorithms for the Minimum Mobile Charger Problem (MinMCP) for two-dimensional (2D) WRSNs and investigated on the design of their recharging routes [92]. Kaikai et al. have explored the transmission energy minimisation issue in a WNSN with the technique of on/off keying (OOK) modulation, and developed the corresponding Minimum Transmission Energy (MTE) coding solutions with the considerations of code rate constraint and code word length constraint [93].

\section{Challenges of Energy Management in RFID Sensor Net- works}

The combination of RFID and WSN is an inevitable advancement in IoT research that will result in a high level of collaboration and technical progress. The goal of achieving effective and robust integration of RFIDs and WSNs needs some technical challenges as depicted in Figure 11, to be addressed as proposed by researchers in the literature. A good collision free scheduling needs to be defined for both RFID and WSN for the reduction of possible interferences under heavy network densities [1].

The distribution of devices, techniques and accessing procedures that can be employed for a wider range of applications is also one of the vital initiatives towards mitigating the issues of integrating RFID and WSN. The factors of cost effective material and most efficient manufacturing processes are also held accountable for the successful integration of RFID and WSN. Hai et al. [2] have presented a discussion on new challenges such as the use of multihop networks to maximise the read range of passive RFID networks and ubiquitous deployment of RFID sensor networks under heavy network densities. The simulators that combine both RFID and WSN also play a prominent role for the development of integrated RSN systems in the field of pervasive computing. The integration of RFIDs and WSNs is a yet to surface and premature technology in the IoT realm. So far, the potential drawbacks in promoting such integration are energy conservation, real-time dynamic performance, semantic data cleaning, data filtering, localisation of nodes, anti-collision, bandwidth constraints and authentication for security concerns [8]. Many of the research contributions for integrating RFID and WSN to attain an energy efficient routing mechanism have only concentrated on the efficiency factor rather than the demand for gaining decisive and realtime interactions. The deployment of huge quantity of RFID readers in the same place will eventually encourage issues such as cost factors, query processing time and interference. The researchers in [12] have proposed that the major challenges accounting for the integration of RFID and WSN include the necessity for extremely lower energy consumption, energy scarcity of sources and inefficient accuracy of low-power sensors.

The integrated systems should have simulators that are capable of modelling, analysis, performance evaluation and optimisation as analysed by authors in [2] and [12]. Energy efficiency has been a critical issue when embedding RFID and WSN together. ZigBee (IEEE 802.15.4) is considered to be the best configuration scheme for WSN based industrial applications as it satisfies reliability, cost factors and meets the need for lower power consumption. ZigBee has no resemblance pertaining to the aspect of data transmission rate because it was not developed for a high data rate duty cycle from each connected node. ZigBee is still an immature technical advancement than the proprietary spread-spectrum solutions [4]. Consequently, there are nil standard based research contributions which can integrate RFID and WSN into one network separately, most probably because of limited research focus, varied research backgrounds and major limitation of higher energy consumption [15]. In order to overcome the significant challenge of limited lifetime of RSN, the two strategies for EM in RSN are $\mathrm{EH}$ and ET.

\section{COMPARISON AND DISCUSSION}

There are significant challenges for communication and routing when RFID and WSN are combined such as localisation of nodes, security breaches, scalability, interoperability and energy scarcity. As shown in Table III, the sensor networks are tabulated according to the applications type and network topology. The type of energy management strategy employed has also been summarised.

The methods by which energy can be managed in sensor networks can be threefold: They are either by mitigating the energy consumed by the nodes, harvesting or storing the energy from sources such as sun/wind/vibration followed by energy recharging and transmission either over dedicated communication channels or wireless medium. These techniques can probably improve the performance of the networks and increase its lifespan, thereby ensuring zero node failures and packet losses. It can be noted evidently that [62], [81], [82], [85], [87], [92] and [94] have focused on energy conservation by combining both RFID and WSN, whereas [69], [70], [83], [84], [86], [88], [89], [93] and [95] 


\begin{tabular}{|c|c|c|c|c|c|c|c|c|c|c|c|c|c|c|}
\hline 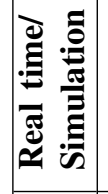 & 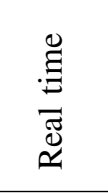 & 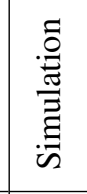 & 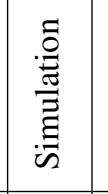 & 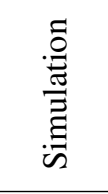 & 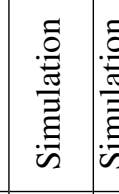 & 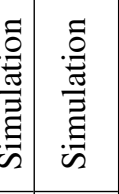 & 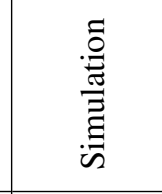 &  & 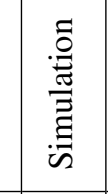 & 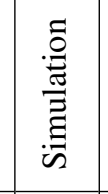 & 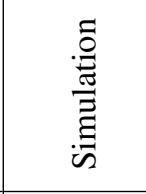 & 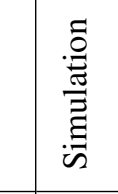 & 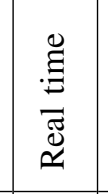 & 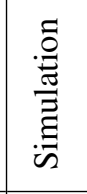 \\
\hline 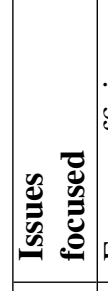 & ) & 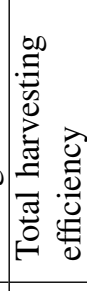 &  & 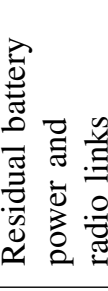 & 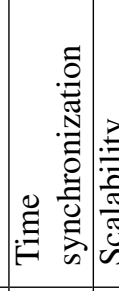 & 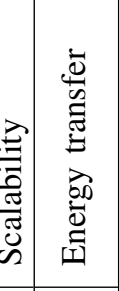 & 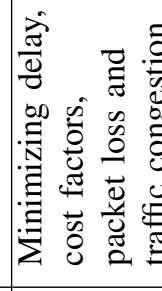 & 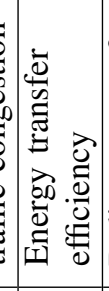 & 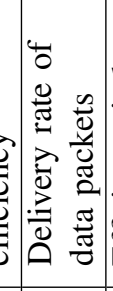 &  & 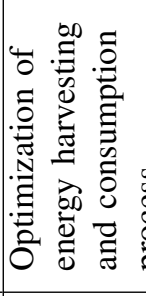 & 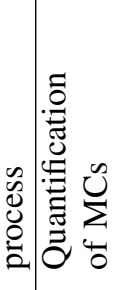 & $\mid$ & 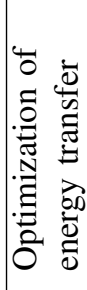 \\
\hline 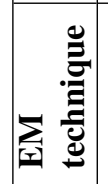 & 䡒 & 丞 & 畄 & 留 & 媓 & 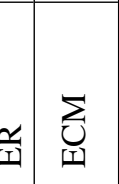 & $\sum_{i}^{\Sigma}$ & 总 & $\sum_{j}^{\Sigma}$ & 竞 & 票 & $\sum_{\text {离 }}$ & $\sum_{i}^{\Sigma}$ & 竞 \\
\hline 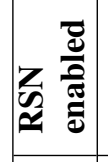 & $\stackrel{\infty}{0}$ & ż & ż & $\stackrel{\nu}{\circ}$ & $\check{z}$ & $\stackrel{8}{8}$ & $\stackrel{0}{0}$ & $\stackrel{8}{z}$ & 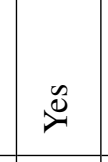 & z & $\stackrel{8}{z}$ & 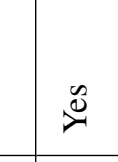 & $\stackrel{\infty}{\infty}$ & ż \\
\hline 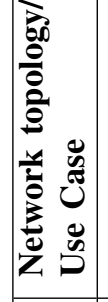 & 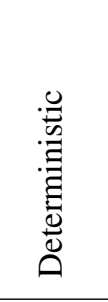 & 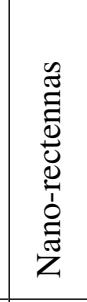 & 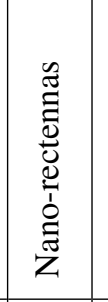 & 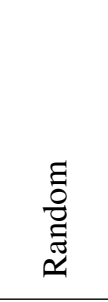 & 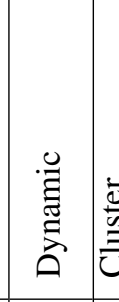 & 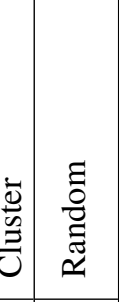 & 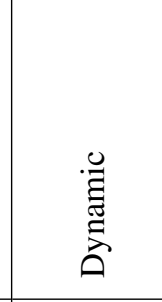 & 离 & 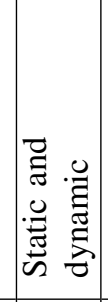 & 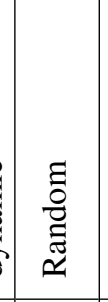 & 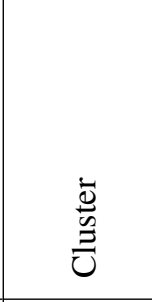 & 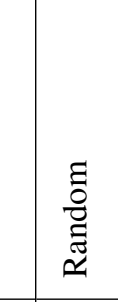 & 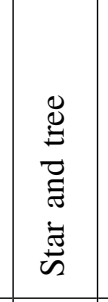 & 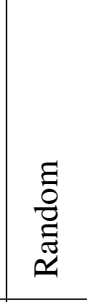 \\
\hline 童 & 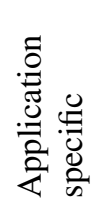 & 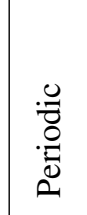 & 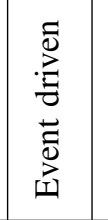 & 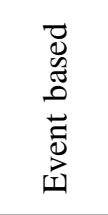 & 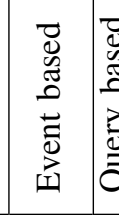 & 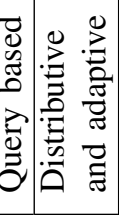 & 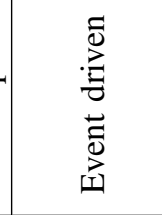 & 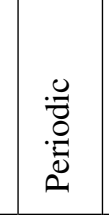 & 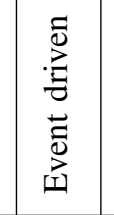 & 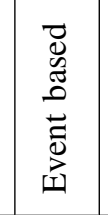 &  & 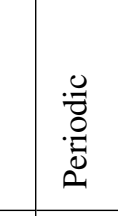 & 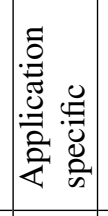 & 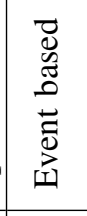 \\
\hline  & 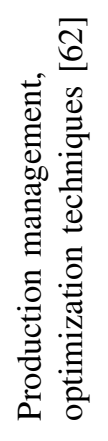 & 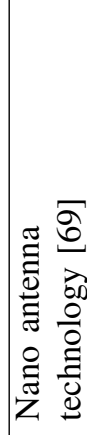 & 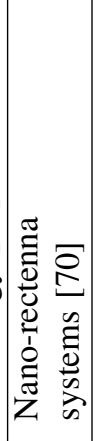 & 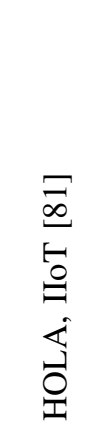 & 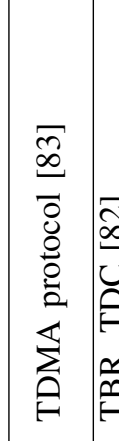 & 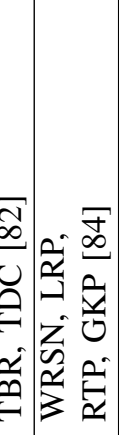 & 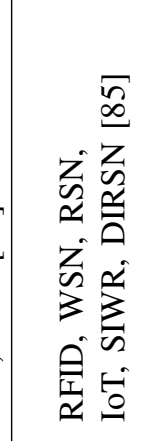 & 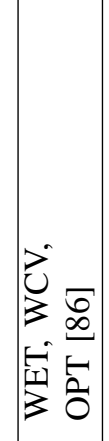 & 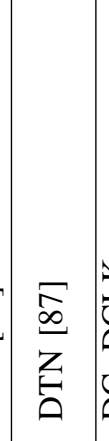 & 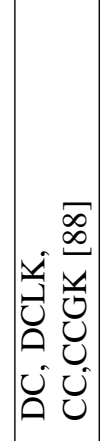 & 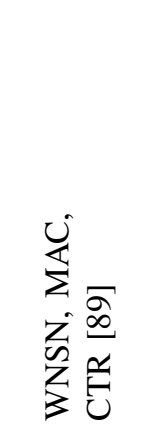 & 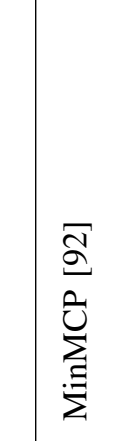 & 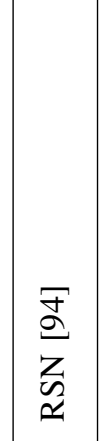 & $\begin{array}{l}\bar{\alpha} \\
\bar{\alpha} \\
z \\
\bar{z} \\
z \\
3\end{array}$ \\
\hline
\end{tabular}


have focused on mitigation of the energy consumed by the communicating nodes for only WSN. Figure 12 and 13 have been depicted on the basis of comparative analysis of all the 95 articles that have been used as references. The tabulation as depicted in Table III had been derived for all articles and with respect to column 4 and 5 of the table. The first pie chart representation in Figure 12 depicts that 68 articles out of 95 are not RSN-enabled and 27 are RSN-enabled. The second graphical representation as in Figure 13 can be interpreted from the statistical analysis that 53 articles have focussed upon mitigation of energy consumption (ECM) as means to manage the energy levels followed by 10 articles on energy recharge (ER), 23 on energy harvesting (EH) and 9 on energy storage (ES) respectively, few of which have been tabulated in Table III. The surveys carried out with regards to sensor networks

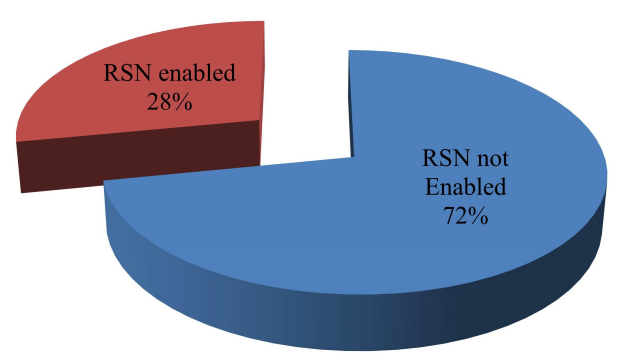

Fig. 12: The percentage of RSN employment among research contributions

down the timeline by the research community have shown that energy consumption plays a vital role for routing and communication. This review article is the first of its kind to have surveyed the core issues of EM for RSN when there are many devices connected to each other over the internet.

Though batteries and capacitors are considered to be the primary energy sources for WSN, its limited lifetime and unpredictability for event-driven nodes makes it possible to face potential drawbacks such as energy scarcity, node failure, packet loss and poor network performance. These reasons eventually lead to either frequent replacement of batteries, sending and receiving recharge requests or periodical eradication of the batteries. Hence, in order to overcome these limitations, energy harvesting from renewable sources of energy have taken a faster pace when compared with existing solutions for RSN communications. This mechanism guarantees a promising solution for the issue of energy scarcity in RSN. This comparative analysis also illustrates the relative issues of energy management that have been solved by the researchers along with the type of experimental validation. The aforementioned illustrations and tabulations drive back the future research directives that need to be addressed for a promising network performance and energy efficient communication for RSN. The authors are working on this aspect of how to manage the harvested RF energy efficiently in order to mitigate node failure and increase the network lifetime. The cooperative solutions that are under development are aimed at providing promising solutions for mobility devices in IoT paradigm by RFEH and transmission of energy over dedicated communication challenges. Further studies in this regard will be carried out to test the feasibility of the solutions

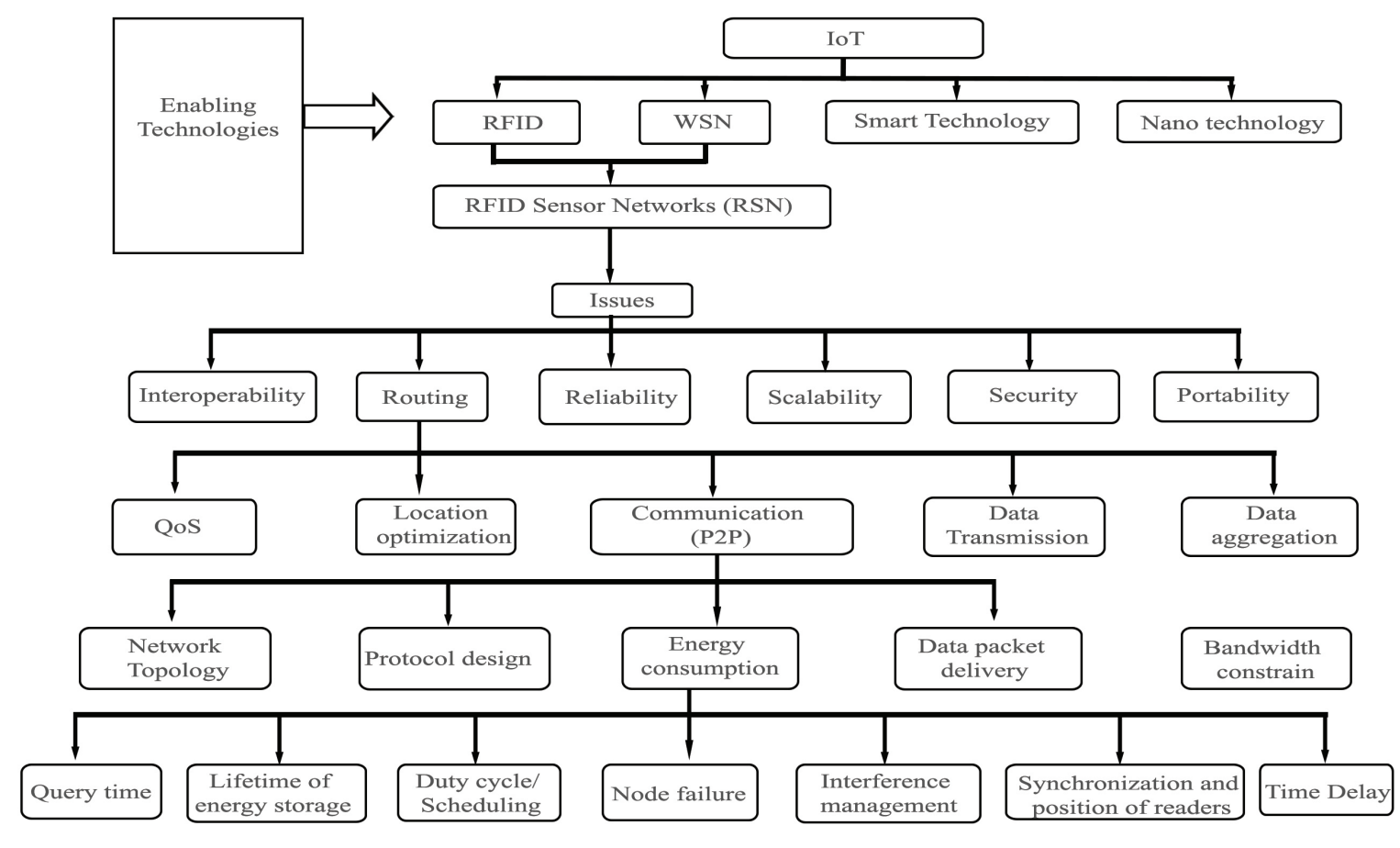

Fig. 11: Taxonomy of RFID Sensor Network Challenges 


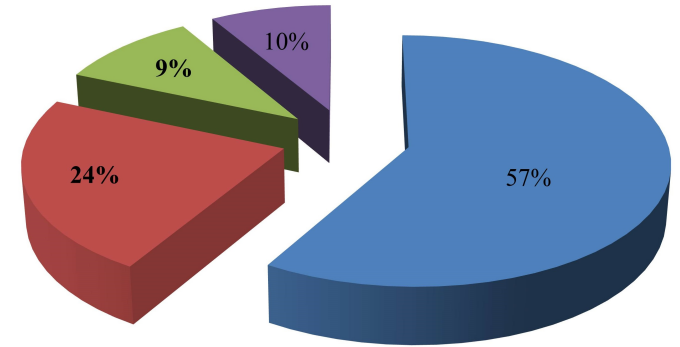

- Mitigation of energy consuption = Energy Harvesting $=$ Energy storage = Energy recharge

Fig. 13: EM techniques and strategies

in comparison with existing solutions for large scale RSN under dynamic network conditions.

\section{IOT - AN OPEN RESEARCH DIRECTIVE}

With IoT being envisioned as the future of smarter technology, it can extensively be utilised for the optimisation of energy when enormous objects are connected over a heterogeneous platform. The aforementioned sections in this paper have clearly stated that when combining RFID and WSN, there is an issue of EM. The evolution of IoT and usage of many devices can help overcome the issue of poor coverage of RFID reader or inefficient read range of passive RFID tags in clustered RSN a ground reality. Hence, the novel concept of Wi-Fi backscattering [74] for instance, can be employed for lowering the energy consumption and thereby, improving the read range of tags. This technique eventually makes EH more dynamic and ET less time-consuming due to smaller energy storage capabilities. But, the issue of reader sensitivity and tag sensitivity in complex integrated systems still needs to be addressed. The design of cross-platform routing protocols for wireless energy transmission through an IoT framework is also seen as the most vital attention seeking research directive. The devices when connected on a shared spectrum can cause interferences over the communication channels. Hence, interference management is also a significant research challenge for EM in RSN and needs to be addressed. The design of novel communication protocols based on IoT that can alleviate the potential drawback of interferences for RF based EM in the wide area of RSN can be articulated as a promising solution. Hence, IoT as a whole, can be foreseen as a promising research directive to overcome the challenges of EM in RSN. Consequently, RF powered cognitive radio network has some of the design issues for RF harvesting which are spectrum sensing, channel access, spectrum management and hand-off. With the advent of spectrum access in a dynamic manner, cognitive radios can improvise the efficiency of the spectrum and capacity of sensor networks. Both spectrum and energy efficiency can be achieved by secondary users through combining RFEH capabilities with cognitive radio network (CRN). Such a network can be termed as RFpowered cognitive radio network. There are recent research contributions which focus upon optimal channel selection, spectrum sensing and bandwidth management. These trade-off based upon spectrum sensing, data transmission and RF energy harvesting in a multi-channel RF-powered CRN are focused upon by the authors in the literature. The detailed description about the research contributions and results achieved is out of the scope of this paper.

\section{CONCLUSION}

In this paper, we have discussed the issue of energy management in an IoT paradigm. The research contributions of integration of key technologies of IoT which are RFID and WSN with their related types, applications and challenges have been outlined through consecutive sections. It was therefore, learnt that energy efficiency is the most vital attention seeking issue that needs to be addressed for RSN. In order to know the concept of energy management in RSN, it is necessary to understand the concept of energy related aspects of RSN in IoT contexts. However, from the discussions in the previous sections of the paper it can be noted that, many researchers have not focused much on the energy management aspect for RSN. Therefore, novel algorithms which take energy conservation into consideration along with energy recharging and optimisation of harvested energy, need to be developed for RSN. Another major attention seeking aspect is developing the simulation tools that will combine both RFID and WSN for energy conservation from renewable sources.

\section{ACKNOWLEDGEMENT}

The authors would like to thank the Sustainable Science Cluster, University of Malaya (Grand Challenge Grant UM.0000007/HRU.GC.SS GC002B-15SUS) for their support. The authors also extend their appreciation to the Deanship of Scientific Research at King Saud University for funding this work through Research Group Number (RG-288).

\section{REFERENCES}

[1] A. Mitrokotsa and C. Douligeris, "Integrated rfid and sensor networks: architectures and applications," RFID and sensor networks: Architectures, protocols, security and integrations, vol. 512, 2009.

[2] H. Liu, M. Bolic, A. Nayak, and I. Stojmenovic, "Taxonomy and challenges of the integration of rid and wireless sensor networks," IEEE network, vol. 22, no. 6, 2008.

[3] A. W. Nagpurkar and S. K. Jaiswal, "An overview of wsn and rfid network integration," in Electronics and Communication Systems (ICECS), 2015 2nd International Conference on. IEEE, 2015, pp. 497-502.

[4] L. Zhang and Z. Wang, "Integration of rfid into wireless sensor networks: architectures, opportunities and challenging problems," in Grid and Cooperative Computing Workshops, 2006. GCCW'06. Fifth International Conference on. IEEE, 2006, pp. 463-469.

[5] M. A. El Khaddar, M. Chraibi, H. Harroud, M. Boulmalf, M. Elkoutbi, and A. Maach, "Modeling and enforcing security and service control policies using flexrfid middleware: Healthcare scenarios," in Science and Information Conference (SAI), 2014. IEEE, 2014, pp. 461-467.

[6] B. Zhang, K. Hu, and Y. Zhu, "Network architecture and energy analysis of the integration of rfid and wireless sensor network," in Control and Decision Conference (CCDC), 2010 Chinese. IEEE, 2010, pp. 13791382.

[7] A. Jedda, M. Khair, and H. T. Mouftah, "Connected coverage for rfid and wireless sensor networks," Procedia Computer Science, vol. 10, pp. 1046-1051, 2012.

[8] L. Wang, L. Da Xu, Z. Bi, and Y. Xu, "Data cleaning for rfid and wsn integration," IEEE transactions on industrial informatics, vol. 10, no. 1, pp. 408-418, 2014.

[9] W. Wang, J. Sung, and D. Kim, "Complex event processing in epc sensor network middleware for both rfid and wsn," in Object Oriented RealTime Distributed Computing (ISORC), 2008 11th IEEE International Symposium on. IEEE, 2008, pp. 165-169. 
[10] J. M. Dupare and N. U. Sambhe, "A novel data cleaning algorithm using rfid and wsn integration," in Innovations in Information, Embedded and Communication Systems (ICIIECS), 2015 International Conference on. IEEE, 2015, pp. 1-5.

[11] F. M. Al-Turjman, A. E. Al-Fagih, and H. S. Hassanein, "A novel costeffective architecture and deployment strategy for integrated rfid and wsn systems," in Computing, Networking and Communications (ICNC), 2012 International Conference on. IEEE, 2012, pp. 835-839.

[12] H. Liu, M. Bolic, A. Nayak, and I. Stojmenovi, Integration of RFID and wireless sensor networks. World Scientific Publishing Company: Toh Tuck, Singapore, 2009.

[13] J. Mitsugi, T. Inaba, B. Pátkai, L. Theodorou, J. Sung, T. S. Lopez, D. Kim, D. McFarlane, H. Hada, Y. Kawakita et al., "Architecture development for sensor integration in the epcglobal network," White Paper WPSWNET-018, Auto-ID Labs, 2007.

[14] U. K. Vishwakarma and R. Shukla, "Wsn and rfid: Differences and integration," International Journal of Advanced Research in Electronics and Communication Engineering, vol. 2, no. 9, pp. 778-780, 2013.

[15] J. Sung, T. S. Lopez, and D. Kim, "The epc sensor network for rfid and wsn integration infrastructure," in Pervasive Computing and Communications Workshops, 2007. PerCom Workshops' 07. Fifth Annual IEEE International Conference on. IEEE, 2007, pp. 618-621.

[16] A. Rida, V. Lakafosis, R. Vyas, M. M. Tentzeris, and S. Nikolaou, "Review of technologies for low-cost integrated sensors," in RFIDTechnologies and Applications (RFID-TA), 2011 IEEE International Conference on. IEEE, 2011, pp. 513-520.

[17] S. K. Gupta and P. Sinha, "Overview of wireless sensor network: A survey," Telos, vol. 3, no. $15 \mu \mathrm{W}$, p. $38 \mathrm{~mW}, 2014$.

[18] H. Yang and S.-H. Yang, "Rfid sensor network network architectures to integrate rfid, sensor and wsn," Measurement and Control, vol. 40, no. 2, pp. 56-59, 2007.

[19] A. G. Ruzzelli, R. Jurdak, and G. M. O'Hare, "On the rfid wake-up impulse for multi-hop sensor networks," in The 1st ACM Workshop on Convergence of RFID and Wireless Sensor Networks and their Applications (SenseID) at the 5th ACM International Conference on Embedded Networked Sensor Systems (ACM SenSys 2007), Sydney, Australia, November 04-09, 2007, 2007.

[20] X. Shi, S. Su, and Q. Xiong, "The integration of wireless sensor networks and rfid for pervasive computing," in Computer Sciences and Convergence Information Technology (ICCIT), 2010 5th International Conference on. IEEE, 2010, pp. 67-72.

[21] M. Baghaei-Nejad, Z. Zou, H. Tenhunen, and L.-R. Zheng, "A novel passive tag with asymmetric wireless link for rfid and wsn applications," in Circuits and Systems, 2007. ISCAS 2007. IEEE International Symposium on. IEEE, 2007, pp. 1593-1596.

[22] S. Hussain, S. Schaffner, and D. Moseychuck, "Applications of wireless sensor networks and rfid in a smart home environment," in Communication Networks and Services Research Conference, 2009. CNSR'09. Seventh Annual. IEEE, 2009, pp. 153-157.

[23] R. Jedermann, C. Behrens, D. Westphal, and W. Lang, "Applying autonomous sensor systems in logisticscombining sensor networks, rfids and software agents," Sensors and Actuators A: Physical, vol. 132, no. 1 , pp. 370-375, 2006.

[24] R. Badia-Melis, J. Garcia-Hierro, L. Ruiz-Garcia, T. Jiménez-Ariza J. I. R. Villalba, and P. Barreiro, "Assessing the dynamic behavior of wsn motes and rfid semi-passive tags for temperature monitoring," Computers and Electronics in Agriculture, vol. 103, pp. 11-16, 2014

[25] A. Nasir and B.-H. Soong, "Environsense: An integrated system for urban sensing using rfid based wsn's," in TENCON 2009-2009 IEEE Region 10 Conference. IEEE, 2009, pp. 1-5.

[26] R. Sharma, P. Singh, and R. Saini, "Notice of violation of ieee publication principles evolution in rfid credit card security by using wireless sensing network," in Management of e-Commerce and e-Government (ICMeCG), 2011 Fifth International Conference on. IEEE, 2011, pp. 229-231.

[27] S. Mirshahi, A. Akbari, and S. Uysal, "Implementation of structural health monitoring based on rfid and wsn," in Electrical and Computer Engineering (CCECE), 2015 IEEE 28th Canadian Conference on. IEEE, 2015, pp. 1318-1323.

[28] Y.-I. Kim, B.-J. Yi, J.-J. Song, J.-H. Shin, and J.-I. Lee, "Implementing a prototype system for power facility management using rfid/wsn," International Journal of Applied Mathematics and Computer Sciences, vol. 2, no. 2, pp. 70-75, 2006.

[29] L. Mainetti, L. Palano, L. Patrono, M. L. Stefanizzi, and R. Vergallo, "Integration of rfid and wsn technologies in a smart parking system," in Software, Telecommunications and Computer Networks (SoftCOM), 2014 22nd International Conference on. IEEE, 2014, pp. 104-110.
[30] S. Ahmed and M. Y. A. Raja, "Integration of wireless sensor network with medical service provider for ubiquitous e-healthcare," in High Capacity Optical Networks and Enabling Technologies (HONET), 2012 9th International Conference on. IEEE, 2012, pp. 120-126.

[31] D. P. Pereira, W. R. A. Dias, M. de Lima Braga, R. da Silva Barreto, C. M. S. Figueiredo, and V. Brilhante, "Model to integration of rfid into wireless sensor network for tracking and monitoring animals," in Computational Science and Engineering, 2008. CSE'08. 11th IEEE International Conference on. IEEE, 2008, pp. 125-131.

[32] S. Mejjaouli and R. F. Babiceanu, "Rfid-wireless sensor networks integration: Decision models and optimization of logistics systems operations," Journal of Manufacturing Systems, vol. 35, pp. 234-245, 2015.

[33] C. Zulkifli and N. Noor, "Wireless sensor network and internet of things (iot) solution in agriculture." Pertanika Journal of Science \& Technology, vol. 25 , no. $1,2017$.

[34] F. Iannello, O. Simeone, and U. Spagnolini, "Energy management policies for passive rfid sensors with rf-energy harvesting," in Соттиnications (ICC), 2010 IEEE International Conference on. IEEE, 2010, pp. $1-6$.

[35] X. Lu, P. Wang, D. Niyato, D. I. Kim, and Z. Han, "Wireless networks with rf energy harvesting: A contemporary survey," IEEE Communications Surveys \& Tutorials, vol. 17, no. 2, pp. 757-789, 2015.

[36] T. H. Dinh, D. Niyato, P. Wang, D. I. Kim, and L. Le, "Optimal data scheduling and admission control for backscatter sensor networks," IEEE Transactions on Communications, 2017.

[37] C. M. Angelopoulos, S. Nikoletseas, T. P. Raptis, C. Raptopoulos, and F. Vasilakis, "Efficient energy management in wireless rechargeable sensor networks," in Proceedings of the 15th ACM international conference on Modeling, analysis and simulation of wireless and mobile systems. ACM, 2012, pp. 309-316.

[38] J. Shi, X. Wei, and W. Zhu, "An efficient algorithm for energy management in wireless sensor networks via employing multiple mobile sinks," International Journal of Distributed Sensor Networks, vol. 2016, 2016.

[39] S. Li, L. Da Xu, and X. Wang, "Compressed sensing signal and data acquisition in wireless sensor networks and internet of things," IEEE Transactions on Industrial Informatics, vol. 9, no. 4, pp. 2177-2186, 2013.

[40] A. P. Sample, D. J. Yeager, P. S. Powledge, A. V. Mamishev, and J. R. Smith, "Design of an rfid-based battery-free programmable sensing platform," IEEE Transactions on Instrumentation and Measurement, vol. 57, no. 11, pp. 2608-2615, 2008.

[41] P. Kamalinejad, C. Mahapatra, Z. Sheng, S. Mirabbasi, V. C. Leung, and Y. L. Guan, "Wireless energy harvesting for the internet of things," IEEE Communications Magazine, vol. 53, no. 6, pp. 102-108, 2015.

[42] G. Abdul-Salaam, A. H. Abdullah, M. H. Anisi, A. Gani, and A. Alelaiwi, "A comparative analysis of energy conservation approaches in hybrid wireless sensor networks data collection protocols," Telecoттиnication Systems, vol. 61, no. 1, pp. 159-179, 2016.

[43] G. Anastasi, M. Conti, M. Di Francesco, and A. Passarella, "Energy conservation in wireless sensor networks: A survey," Ad hoc networks, vol. 7, no. 3, pp. 537-568, 2009.

[44] R. J. Vullers, R. Van Schaijk, H. J. Visser, J. Penders, and C. Van Hoof, "Energy harvesting for autonomous wireless sensor networks," IEEE Solid-State Circuits Magazine, vol. 2, no. 2, pp. 29-38, 2010.

[45] F. Yang, L. Du, W. Chen, J. Li, Y. Wang, and D. Wang, "Hybrid energy harvesting for condition monitoring sensors in power grids," Energy, vol. 118, pp. 435-445, 2017.

[46] P. Zhang, G. Xiao, and H.-P. Tan, "Clustering algorithms for maximizing the lifetime of wireless sensor networks with energy-harvesting sensors," Computer Networks, vol. 57, no. 14, pp. 2689-2704, 2013.

[47] K. Niotaki, A. Collado, A. Georgiadis, S. Kim, and M. M. Tentzeris, "Solar/electromagnetic energy harvesting and wireless power transmission," Proceedings of the IEEE, vol. 102, no. 11, pp. 1712-1722, 2014.

[48] M. Arrawatia, M. S. Baghini, and G. Kumar, "Differential microstrip antenna for rf energy harvesting," IEEE Transactions on Antennas and Propagation, vol. 63, no. 4, pp. 1581-1588, 2015.

[49] X. Lu, P. Wang, D. Niyato, and Z. Han, "Resource allocation in wireless networks with rf energy harvesting and transfer," IEEE Network, vol. 29, no. 6 , pp. $68-75,2015$.

[50] A. E. Abdulhadi and R. Abhari, "Multiport uhf rfid-tag antenna for enhanced energy harvesting of self-powered wireless sensors," IEEE Transactions on Industrial Informatics, vol. 12, no. 2, pp. 801-808, 2016.

[51] F. K. Shaikh and S. Zeadally, "Energy harvesting in wireless sensor networks: A comprehensive review," Renewable and Sustainable Energy Reviews, vol. 55, pp. 1041-1054, 2016. 
[52] D. J. Pérez, R. G. Gómez, and J. T. Gómez, "A survey of energy harvesting circuits: research issues and challenges.” Revista Telem@ tica, vol. 15, no. 2, pp. 73-90, 2016.

[53] W.-C. Shih, P. H. Chou, and W.-T. Chen, "A batteryless beacon based on dual ism-band rf harvesting with solar-biasing current," in Proceedings of the 4th International Workshop on Energy Harvesting and EnergyNeutral Sensing Systems. ACM, 2016, pp. 7-12.

[54] M. H. Anisi, G. Abdul-Salaam, M. Y. I. Idris, A. W. A. Wahab, and I. Ahmedy, "Energy harvesting and battery power based routing in wireless sensor networks," Wireless Networks, pp. 1-18, 2015.

[55] M. H. Anisi, J. Rezazadeh, and M. Dehghan, "Feda: Fault-tolerant energy-efficient data aggregation in wireless sensor networks," in Software, Telecommunications and Computer Networks, 2008. SoftCOM 2008. 16th International Conference on. IEEE, 2008, pp. 188-192.

[56] M. H. Anisi, G. Abdul-Salaam, and A. H. Abdullah, "A survey of wireless sensor network approaches and their energy consumption for monitoring farm fields in precision agriculture," Precision Agriculture, vol. 16, no. 2, pp. 216-238, 2015.

[57] G. Han, A. Qian, J. Jiang, N. Sun, and L. Liu, "A grid-based joint routing and charging algorithm for industrial wireless rechargeable sensor networks," Computer Networks, vol. 101, pp. 19-28, 2016.

[58] I. Bisio, A. Sciarrone, and S. Zappatore, "A new asset tracking architecture integrating rfid, bluetooth low energy tags and ad hoc smartphone applications," Pervasive and Mobile Computing, vol. 31, pp. 79-93, 2016.

[59] A. R. Devidas, S. R. George, and M. V. Ramesh, "A system for energy conservation through personalized learning mechanism," in Smart Cities and Green ICT Systems (SMARTGREENS), 2015 International Conference on. IEEE, 2015, pp. 1-7.

[60] S. Ganguly, "An energy efficient evo-fuzzy sleep scheduling protocol for stationary target coverage in wireless sensor networks," Journal of Wireless Sensor Network, vol. 3, no. 1, 2016.

[61] A. Z. Kausar, A. W. Reza, M. U. Saleh, and H. Ramiah, "Energizing wireless sensor networks by energy harvesting systems: Scopes, challenges and approaches," Renewable and Sustainable Energy Reviews, vol. 38, pp. 973-989, 2014.

[62] F. Shrouf and G. Miragliotta, "Energy management based on internet of things: practices and framework for adoption in production management," Journal of Cleaner Production, vol. 100, pp. 235-246, 2015.

[63] X. Fafoutis, T. Sørensen, and J. Madsen, "Energy harvesting-wireless sensor networks for indoors applications using ieee 802.11," Procedia Computer Science, vol. 32, pp. 991-996, 2014.

[64] R. M. Ferdous, A. W. Reza, and M. F. Siddiqui, "Renewable energy harvesting for wireless sensors using passive rfid tag technology: A review," Renewable and Sustainable Energy Reviews, vol. 58, pp. 1114 1128, 2016.

[65] D. Marrero, E. Macías, Á. Suárez, J. A. Santana, and V. Mena, "Energy saving in smart city wireless backbone network for environment sensors," Mobile Networks and Applications, pp. 1-12, 2016.

[66] S. Sudevalayam and P. Kulkarni, "Energy harvesting sensor nodes: Survey and implications," IEEE Communications Surveys \& Tutorials, vol. 13, no. 3, pp. 443-461, 2011.

[67] F. Akhtar and M. H. Rehmani, "Energy replenishment using renewable and traditional energy resources for sustainable wireless sensor networks: A review," Renewable and Sustainable Energy Reviews, vol. 45, pp. 769-784, 2015.

[68] G. Piro, G. Boggia, and L. A. Grieco, "On the design of an energyharvesting protocol stack for body area nano-networks," Nano Cоттиnication Networks, vol. 6, no. 2, pp. 74-84, 2015.

[69] Z. Ma, X. Zheng, G. A. Vandenbosch, and V. Moshchalkov, "On the efficiency of solar energy harvesting with nano-dipoles," in Antennas and Propagation (EuCAP), 2013 7th European Conference on. IEEE, 2013, pp. 2856-2859.

[70] Z. Ma and G. A. Vandenbosch, "Optimal solar energy harvesting efficiency of nano-rectenna systems," Solar Energy, vol. 88, pp. 163174, 2013.

[71] J.-D. Park, H. Lee, and M. Bond, "Uninterrupted thermoelectric energy harvesting using temperature-sensor-based maximum power point tracking system," Energy Conversion and Management, vol. 86, pp. 233-240, 2014.

[72] P. Nintanavongsa, M. Y. Naderi, and K. R. Chowdhury, "A dual-band wireless energy transfer protocol for heterogeneous sensor networks powered by rf energy harvesting," in Computer Science and Engineering Conference (ICSEC), 2013 International. IEEE, 2013, pp. 387-392.

[73] Y. Chu, S. Kosunalp, P. D. Mitchell, D. Grace, and T. Clarke, "Application of reinforcement learning to medium access control for wireless sensor networks," Engineering Applications of Artificial Intelligence, vol. 46, pp. 23-32, 2015

[74] B. Kellogg, A. Parks, S. Gollakota, J. R. Smith, and D. Wetherall, "Wi-fi backscatter: Internet connectivity for rf-powered devices," $A C M$ SIGCOMM Computer Communication Review, vol. 44, no. 4, pp. 607618, 2015.

[75] Z. Hadzi-Velkov, N. Zlatanov, and R. Schober, "Multiple-access fading channel with wireless power transfer and energy harvesting," IEEE Communications Letters, vol. 18, no. 10, pp. 1863-1866, 2014.

[76] X. Lu, I. Flint, D. Niyato, N. Privault, and P. Wang, "Performance analysis of simultaneous wireless information and power transfer with ambient rf energy harvesting," in Wireless Communications and Networking Conference (WCNC), 2015 IEEE. IEEE, 2015, pp. 1303-1308.

[77] J. Blanco, F. Bolos, A. Collado, and A. Georgiadis, "Rf-energy harvesting and wireless power transfer efficiency from digitally modulated signals," in Microwave Symposium (MMS), 2015 IEEE 15th Mediterranean. IEEE, 2015, pp. 1-4.

[78] A. N. Parks, A. Liu, S. Gollakota, and J. R. Smith, "Turbocharging ambient backscatter communication," in ACM SIGCOMM Computer Communication Review, vol. 44, no. 4. ACM, 2014, pp. 619-630.

[79] M. Buettner, B. Greenstein, and D. Wetherall, "Dewdrop: an energyaware runtime for computational rfid," in Proc. USENIX NSDI, 2011, pp. 197-210.

[80] L. Fu, P. Cheng, Y. Gu, J. Chen, and T. He, "Optimal charging in wireless rechargeable sensor networks," IEEE Transactions on Vehicular Technology, vol. 65, no. 1, pp. 278-291, 2016.

[81] K. Dhondge, R. Shorey, and J. Tew, "Hola: Heuristic and opportunistic link selection algorithm for energy efficiency in industrial internet of things (iiot) systems," in Communication Systems and Networks (COMSNETS), 2016 8th International Conference on. IEEE, 2016, pp. $1-6$.

[82] I. Farris, L. Militano, A. Iera, A. Molinaro, and S. C. Spinella, "Tagbased cooperative data gathering and energy recharging in wide area rfid sensor networks," Ad Hoc Networks, vol. 36, pp. 214-228, 2016.

[83] Z. Chen, D. Li, Y. Huang, and C. Tang, "Event-triggered communication for distributed time synchronization in wsns," in Control Conference (CCC), 2015 34th Chinese. IEEE, 2015, pp. 7789-7794.

[84] C. M. Angelopoulos, S. Nikoletseas, and T. P. Raptis, "Wireless energy transfer in sensor networks with adaptive, limited knowledge protocols," Computer Networks, vol. 70, pp. 113-141, 2014.

[85] F. M. Al-Turjman, A. E. Al-Fagih, W. M. Alsalih, and H. S. Hassanein, "A delay-tolerant framework for integrated rsns in iot," Computer Communications, vol. 36, no. 9, pp. 998-1010, 2013.

[86] L. Xie, Y. Shi, Y. T. Hou, W. Lou, H. D. Sherali, H. Zhou, and S. F. Midkiff, "A mobile platform for wireless charging and data collection in sensor networks," IEEE Journal on Selected Areas in Communications, vol. 33, no. 8, pp. 1521-1533, 2015.

[87] D. Niyato, P. Wang, H.-P. Tan, W. Saad, and D. I. Kim, "Cooperation in delay-tolerant networks with wireless energy transfer: Performance analysis and optimization," IEEE Transactions on Vehicular Technology, vol. 64, no. 8, pp. 3740-3754, 2015.

[88] A. Madhja, S. Nikoletseas, and T. P. Raptis, "Distributed wireless power transfer in sensor networks with multiple mobile chargers," Computer Networks, vol. 80, pp. 89-108, 2015.

[89] P. Wang, J. M. Jornet, M. A. Malik, N. Akkari, and I. F. Akyildiz, "Energy and spectrum-aware mac protocol for perpetual wireless nanosensor networks in the terahertz band," Ad Hoc Networks, vol. 11, no. 8, pp. 2541-2555, 2013

[90] S. Padakandla, K. Prabuchandran, and S. Bhatnagar, "Energy sharing for multiple sensor nodes with finite buffers," IEEE Transactions on Communications, vol. 63, no. 5, pp. 1811-1823, 2015.

[91] L. Huang, W. Wang, and S. Shen, "Energy-efficient coding for electromagnetic nanonetworks in the terahertz band," Ad Hoc Networks, vol. 40, pp. 15-25, 2016.

[92] H. Dai, X. Wu, G. Chen, L. Xu, and S. Lin, "Minimizing the number of mobile chargers for large-scale wireless rechargeable sensor networks," Computer Communications, vol. 46, pp. 54-65, 2014.

[93] K. Chi, Y.-h. Zhu, X. Jiang, and X. Tian, "Optimal coding for transmission energy minimization in wireless nanosensor networks," Nano Communication Networks, vol. 4, no. 3, pp. 120-130, 2013

[94] W. Ding, "Minimizing wsn energy and cost by embedding rfid tags," in Global High Tech Congress on Electronics (GHTCE), 2013 IEEE. IEEE, 2013, pp. 50-55.

[95] H. N. Rafsanjani, C. R. Ahn, and M. Alahmad, "A review of approaches for sensing, understanding, and improving occupancy-related energy-use behaviors in commercial buildings," Energies, vol. 8, no. 10, pp. 10996 $11029,2015$. 


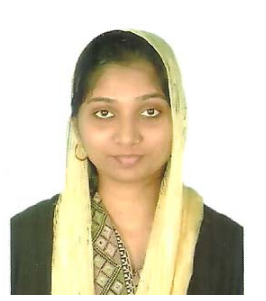

Shaik Shabana Anjum is a $\mathrm{PhD}$ candidate at the Department of Computer System and Technology, Faculty of Computer Science and Information Technology, University of Malaya. She had received her Bachelor degree in Computer Science and Engineering during the year 2010 and Masters in Software Engineering during 2012 from Anna University, Chennai, India. Her research interests include Internet of Things, Internet of Energy, Wireless Sensor Networks, RFID and energy harvesting.

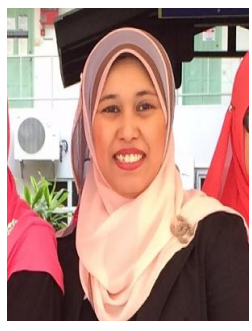

Rafidah Md Noor received her BIT from University Utara Malaysia in 1998, and MSc in Computer Science from University Technology Malaysia in 2000, and $\mathrm{PhD}$ in Computing from Lancaster University, United Kingdom in 2010. She is currently an Associate Professor with the Department of Computer System and Technology, Faculty of Computer Science and Information Technology, University of Malaya. Rafidah's research is related to a field of transportation system in computer science research domain.Her research interests include vehicular networks, network mobility, Quality of Service and Quality of Experience. She has several collaborators from China, Taiwan, South Korea, France, Australia, and United Kingdom who are willing to support in providing excellent research outputs.

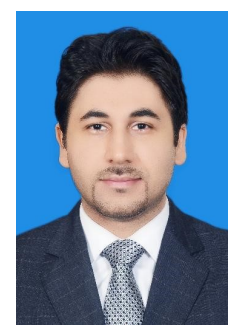

Mohammad Hossein Anisi is with the School of Computer Science and Electronic Engineering, University of Essex, United Kingdom. He received his $\mathrm{PhD}$ from Universiti Teknologi Malaysia (UTM) while being awarded as the best postgraduate student. He has worked as senior lecturer at the Faculty of Computer Science and Information Technology, University of Malaya. His research interests lie in the area of Internet of Things, wireless sensor networks and their applications, Mobile Ad hoc networks, and intelligent transportation systems. He has also collaborated actively with researchers in several other disciplines of computer science. He has published several papers in high quality journals and conferences. He is associate editor of the Ad Hoc and Sensor Wireless Networks (SCIE) and the KSII Transactions on Internet and Information Systems (SCIE) journals. He is also active member of IEEE, ACM, International Association of Engineers (IAENG) and Institute of Research Engineers and Doctors (the IRED).

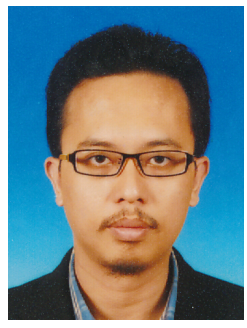

Ismail Ahmedy is one of the academic members in Department of Computer System and Technology, University of Malaya since 2007. He received his BSc (Computer Science) from University Teknologi Malaysia in 2006. After completing his study, he has been granted a full scholarship to pursuing his studies in Master degree where he receives his MSc (Computer Science) from University of Queensland, Australia. He obtained his $\mathrm{PhD}$ in Universiti Teknologi Malaysia in Wireless Networks System specializing in wireless sensor networks in routing

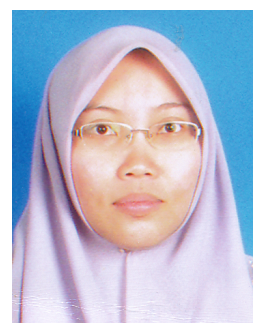

Fazidah Othman is a lecturer at the Department of Computer System and Technology, Faculty of Computer Science and Information Technology, University of Malaya. Her research interest includes Computer Communication, Digital Forensic, Steganography, Network Security, Public Key Infrastructure and Biometrics.

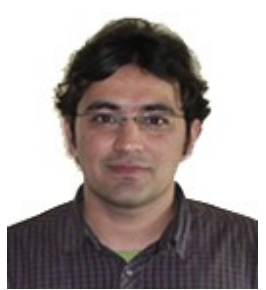

Muhammad Alam holds a $\mathrm{PhD}$ degree in computer science from University of Aveiro, Portugal (201314). In 2009, he joined the Instituto de Telecomunicaes - Aveiro (Portugal) as researcher and completed his $\mathrm{PhD}$ from University of Aveiro with a specialization in Inter Layer and Cooperative Design Strategies for Green Mobile Networks. Currently, he is working as senior researcher at Instituto de Telecomunicaes and participating in European Union and Portuguese government funded projects. His research interests include IoT, Real-time wireless communication, 5G, Vehicular networks, Context-aware systems and Radio resource management in next generation wireless networks. He is the editor of Book "Intelligent Transportation Systems, Dependable Vehicular Communications for Improved Road Safety". He is the author of several journal and conference publications as well as book chapters. He is also the TPC member and reviewer for a number of reputed conferences, journals, and magazines. He is IEEE and IEEE IES member. He served as general co-chair of future $5 \mathrm{~V}$ conference and also served as session chairs in a number of reputed conferences such as IEEE IECON 2016, IEEE WFCS 2016, IEEE ITSC 2015. He also provided his services as guest editor to several journals.

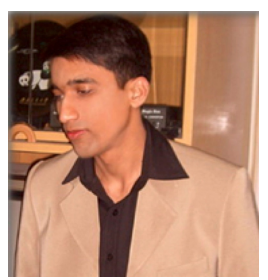

Muhammad Khurran Khan is currently working as a Full Professor at the Center of Excellence in Information Assurance (CoEIA), King Saud University, Kingdom of Saudi Arabia. He is one of the founding members of CoEIA and has served as the Manager at Research and Development from March 2009 to March 2012. He developed and successfully managed the research program of CoEIA, which transformed the center as one of the best centers of research excellence in Saudi Arabia as well as in the region. Prof. Khurram has published over 250 research papers in the journals and conferences of international repute. In addition, he is an inventor of 10US/PCT patents. He has edited 7 books/proceedings published by Springer-Verlag and IEEE. He has secured several national and international research grants in the domain of information security. His research areas of interest are Cybersecurity, digital authentication, biometrics, multimedia security, and technological innovation management. Prof. Khurram has recently played a leading role in developing 'BS Cybersecurity Degree Program' and 'Higher Diploma in Cybersecurity' at King Saud University. 